\title{
Research Square \\ Destabilization of The Bacterial Interactome Tracks Nutrient Restriction-Induced Dysbiosis in Insect Gut
}

\section{Ramona Marasco}

KAUST: King Abdullah University of Science and Technology

\section{Marco Fusi}

KAUST: King Abdullah University of Science and Technology

\section{Matteo Callegari}

KAUST: King Abdullah University of Science and Technology

Costanza Juker

University of Milan

Francesca Mapelli

University of Milan

\section{Sara Borin}

University of Milan

\section{Sara Savoldelli}

University of Milan

Daniele Daffonchio ( $\nabla$ daniele.daffonchio@kaust.edu.sa )

King Abdullah University of Science and Technology https://orcid.org/0000-0003-0947-925X

\section{Elena Crotti}

University of Milan

\section{Research}

Keywords: Nutrient restriction, Dysbiosis, Bacterial microbiome, Beta-diversity, Dispersion, Black soldier fly, Interactome, Co-occurrence network, Gut, Keystone species

Posted Date: November 13th, 2020

DOI: https://doi.org/10.21203/rs.3.rs-104571/v1

License: (1) This work is licensed under a Creative Commons Attribution 4.0 International License. Read Full License 
1 Destabilization of the bacterial interactome tracks nutrient restriction-induced dysbiosis in insect

2 gut

3

4 Ramona Marasco ${ }^{1 \#}$, Marco Fusi ${ }^{1 \# \ddagger}$, Matteo Callegari ${ }^{1 \#}$, Costanza Jucker ${ }^{2}$, Francesca Mapelli², Sara

5 Borin $^{2}$, Sara Savoldelli ${ }^{2}$, Daniele Daffonchio ${ }^{1 *}$, and Elena Crotti2 ${ }^{2}$

6

$7 \quad{ }^{1}$ Biological and Environmental Sciences and Engineering Division (BESE), King Abdullah University

8 of Science and Technology (KAUST), Thuwal 23955-6900, Kingdom of Saudi Arabia

$9{ }^{2}$ Department of Food, Environmental and Nutritional Sciences (DeFENS), University of Milano, Milan,

10 Italy

11

$12 \quad{ }^{\#}$ Equal contributors

$13 *$ Correspondence to Elena Crotti, elena.crotti@unimi.it and Daniele Daffonchio,

14 daniele.daffonchio@kaust.edu.sa

15 Present Address: School of Applied Sciences, Edinburgh Napier University, Edinburgh, UK

16 


\section{Abstract}

19 Background. Stress affects host growth and development and can induce changes in the gut microbiome, 20 commonly defined as dysbiosis. Dysbiosis has been proposed to affect community beta-diversity and 21 within-beta-diversity (community dispersion). As abiotic and biotic stresses, nutrient restriction (NR) 22 also impairs host fitness and results in dysbiosis. However, NR does not introduce overt negative 23 effectors or selectors, such as toxic compounds, pathogens, or parasites, resulting in its role as a 24 determinant of beta-diversity changes being questioned. We hypothesize that following NR, gut dysbiosis is reflected via changes in networking properties of the microbiome rather than via variation in its beta-diversity and/or dispersion. To test our hypothesis, we fed the black soldier fly, Hermetia illucens, a nutritionally versatile polyphagous insect, with two NR diets and a control full-nutrient (FN) diet. Then, we assessed the effects of NR on insect growth and development and gut physicochemical conditions to validate the presence of dysbiosis. In addition, we analyzed the bacterial diversity associated with larvae, pupae, and adults via $16 \mathrm{~S}$ rRNA gene sequencing to assess the role of NR on the composition, structure, and stability of the bacterial communities.

Results. NR strongly affected insect growth and development, inducing significant changes in the physiochemical conditions of the larval gut. Further, diet-dependent differences in bacterial composition - expected in holometabolous/polyphagous insects - were observed, with enrichment in diet-specific keystone bacterial taxa (Bacilli in FN-fed individuals and Clostridia and Gamma- and Alphaproteobacteria in NR-fed individuals), and greater microbiome dispersion in adults but not in larvae and pupae.

Conclusions. While NR establishes alternative stable configurations of the gut microbiome compared with normally fed gut, NR-driven dysbiotic growth performance is considerably reflected in rarefied, less structured, and connected bacterial interactomes than in within beta-diversity changes. 
42 Keywords: Nutrient restriction, Dysbiosis, Bacterial microbiome, Beta-diversity, Dispersion, Black

43 soldier fly, Interactome, Co-occurrence network, Gut, Keystone species

\section{Background}

In healthy animals, gut microbial communities promote host development, nutrition, growth, and homeostasis favored by compositional and functional diversity [1,2]. Polyphagous animals, who may experience periodical compositional changes in diet, have variable microbiome configurations, which are all defined as normobiotic if they support healthy conditions [2-7]. Considering the conceptual energy landscape [1], a microbiome configuration can shift to another configuration when selective forces are stronger than actual stability-driving forces. Such alternative gut microbiome configurations can be considered neutral for health (i.e., associated with healthy host conditions) when they continue to support resistance to perturbations and resilience [1,2]. However, such normobiotic healthy microbiome configurations may be replaced by those associated with dysbiosis $[1,8]$; when microbiome changes are not able to support resistance to perturbation and lose resilience, they result in functional alterations in host health and fitness [2,9-11].

Diet has been recognized as a factor that reshapes the gut microbiome [4,6,12-15], even to configurations that are associated with disease conditions (i.e., dysbiosis; [1,2,15-18]). The factors driving diet-induced changes in microbiome configurations in the absence of external negative effectors, such as pathogens,

61 parasites, or toxic compounds, are not well understood. Many studies have assessed the effect of specific 62 nutrient contents, including fats, fibers, and vitamins, on the diet [16,17]. For instance, in drosophila, a 63 thiamine-lacking diet impacts offspring development; this impact can be rescued by an acetic acid 64 bacterium, thereby representing a potential dysbiosis-sensitive element of the drosophila microbiome 
65 [18]. While the association between specific diet components and dysbiotic members of the gut 66 microbiome is necessary for a mechanistic understanding of the functional alterations associated with 67 dysbiosis, the conditions altering the microbiome composition and configuration in response to diet 68 changes, particularly in polyphagous animals that rely on complex and variable diets, are elusive. We 69 hypothesize that in such animals - equipped with complex microbiomes, including a large range of commensals [19-21] — the primary driver of the loss of resilience in diet-induced dysbiosis observed under a changed microbiome configuration $[2,22,23]$ is not only associated with changes in diversity, including beta-diversity and dispersion (as proposed by Zaneveld et al. [24]), but primarily with changes in the structure of the bacterial network. In particular, we aimed to assess whether exposure to diet-related stressors determine (i) either a new stable configuration of the gut microbiome or a stochastically-based alteration (i.e., greater microbiome dispersion; [24]) and (ii) either a stable or disrupted interactome network among members of the microbiome.

To test our hypothesis, we used the saprophagous and omnivorous black soldier fly (BSF), Hermetia illucens [25-28]. The nutritional versatility of BSF makes it home to a complex bacterial microbiome, with widespread potential for complex bacterial networking [29]. We compared the growth and development of BSF under two different conditions of strong nutrient restriction (NR), namely fruit and vegetable NR diet (NRF and NRV, respectively), and a normal full-nutrient (FN) diet. The reason for choosing NR as a challenging condition is because it does not introduce overt external negative effectors or selectors of the microbiome but may exert adverse effects on the animals' growth and development as well as modify the physicochemical conditions of the gut [30,31]. We assessed the changes in bacterial diversity and interactome at different stages of the BSF's life cycle (i.e., larvae, pupae, and adult) under the three feeding regimens (FN vs. NRF vs. NRV) to link the observed changes in bacterial composition, structure, and networking properties with the fitness and growth performance of the host. 
NR affects the development and growth performance of the insect. The two NR diets had lower nutrient content (i.e., proteins, lipids, carbohydrates, and fibers) than the control FN diet; however, they had different carbohydrate-to-protein ratios to induce different NRs (namely, NRF > carbohydrates and NRV > proteins and moisture; Figure 1a and Additional file 1). Both NR diets resulted in limited availability of food-energy compared with FN ( $-77 \%$ and $-88 \% \mathrm{kcal} / \mathrm{g}$ in NRF and NRV, respectively), with less calories per day $(-79 \%$ and $-92 \% \mathrm{kcal} /$ day/larvae; Table 1$)$. The energy limitations of NRF and NRV diets significantly induced longer larval-prepupal development times $\left(\mathrm{F}_{2,15}=32.5, p<0.0001\right.$; Table 1) and lower larval growth rates (Figure 1b), with a final significant reduction in insect body size at each stage of the BSF life cycle (weight, larvae: $\mathrm{F}_{2,15}=47, p=0.0002$; pupae: $\mathrm{F}_{2,357}=357, p<0.0001$; adult: $\mathrm{F}_{2,717}=631.6, p<0.0001$; Figures $1 \mathrm{c}-\mathrm{e}$ and length, pupae: $\mathrm{F}_{2,177}=168, p<0.0001 ;$ adult: $\mathrm{F}_{2,357}=$ 253, $p<0.0001$; Additional file 2; e.g., Additional file 3). These growth performances (i.e., weight) negatively correlated with moisture and positively correlated with all other diet components (i.e., protein, lipid, carbohydrate, and fiber; Additional file 4).

The waste reduction and bioconversion of experimental substrates by BSF larvae significantly differed between FN and NR diets (waste reduction index (WRI): $\mathrm{F}_{2,6}=2162, p<0.0001$; substrate reduction: $\mathrm{F}_{2,6}=140, p<0.0001$; efficiency of conversion of digested food (ECD): $\mathrm{F}_{2,6}=538.4, p<0.0001$; Table 1). For instance, larvae more efficiently reduced FN substrates (highest WRI and ECI), with 2- and 5fold less substrate consumption compared with those fed with NRV and NRF diets, respectively (Table 1). Notably, NR diets had no effect on larval survival to the prepupal stage $\left(\mathrm{F}_{2,6}=2.27, p=0.18\right)$ but had significantly less adult emergence $\left(\mathrm{F}_{2,6}=87, p<0.0001 ; \mathrm{NRF}=-13 \%\right.$ and $\left.\mathrm{NRV}=-40 \%\right)$ and survival $111\left(\mathrm{~F}_{2,6}=66.5, p<0.0001 ; \mathrm{NRF}=-8 \%\right.$ and $\mathrm{NRV}=-36 \%$; Table 1$)$. 
112 Considering the post-feeding conditions of BSF pupae [32] and the morphogenetic events affecting BSF

113 adults [33], we determined the physicochemical conditions of oxygen partial pressure, $\mathrm{pH}$, and redox

114 potential only of larval gut. Interestingly, among all the examined gut parts, significant changes in the

115 examined parameters were only observed in the midgut (Additional file Figure 5). As the midgut lacks

116 an exoskeletal lining owing to its endodermal origin, it is the primary site of digestion and nutrient

117 absorption [34,35]. For instance, while oxygen concentration was close to zero in the gut lumen of FN-

118 fed individuals, a higher variability was measured in the midgut of NR-fed larvae $\left(\mathrm{F}_{2,16}=4.29, p=0.032\right)$,

119 with oxygen concentrations reaching up to $20 \mu \mathrm{mol} / \mathrm{L}$ in NRV-fed insects (Additional file 5). Although

120 the increment in oxygen concentration in NR midguts was minimal, such changes can have detrimental

121 effects on larval growth and molting [36,37]. We also detected changes in gut lumen $\mathrm{pH}$, with diet having

122 a significant effect in the distal portions of the gut (midgut: $\mathrm{F}_{2,18}=11.41, p=0.0006$ and hindgut: $\mathrm{F}_{2,18}=$

$12312.41, p=0.0004$; Additional file 5); we recorded a more alkaline $\mathrm{pH}$ in NR-fed larvae (up to 9.1 and

1249.9 in NRF- and NRV-fed larvae, respectively) than in FN-fed larvae (for instance, the midgut in

125 Additional file 5). Such $\mathrm{pH}$ changes might influence nutrient availability by affecting the performance

126 of larval gut enzymes (such as proteases) [38]. Along with $\mathrm{pH}$ and oxygen concentration changes, we

127 also measured differences in redox potential in the larval midgut $\left(\mathrm{F}_{2,17}=4.38, p=0.03\right)$. We recorded

128 significantly lower redox values in the midgut of NR-fed insects (average, 165 and $240 \mathrm{mV}$ in the NRF-

129 and NRV-fed insects, respectively) than in the FN-fed insects (299 mV; Additional file 5), indicating

130 changes in the microbial activity and metabolism in the midgut $[39,40]$.

132 Effect of NR on the bacterial community structure and composition of BSF gut. The effects of NR

133 on the growth and development of BSF as well as on the physicochemical conditions of the gut were also

134 reflected in the structure and composition of gut bacterial communities. At each developmental stage,

135 the bacterial communities associated with NR-fed guts formed distinct clusters (the 16S rRNA gene in 
136 Figures 2a-c and 16S-23S rRNA internal transcribed spacers (ITS) in Additional file 6; statistical analysis

137 in Additional file 7), with the diet factor explaining up to $36 \%$ of the total microbiome diversity

138 (Additional file 8). Among the diet components, the higher concentration of moisture in NR diets, in

139 addition to lower supply of carbohydrates and protein in NRV and NRF, respectively, significantly

140 explained the differences in bacterial communities during BSF development (Additional file 9). Notably,

141 the observed changes in bacterial communities (Bray-Curtis similarity) also significantly correlated with

142 the deterioration in the health of the host (i.e., weight loss; Additional file 10), indicating an NR-driven

143 dysbiosis starting from the initial phase of insect development.

144 Differences in richness (i.e., the number of operational taxonomic units (OTUs); Additional file 11)

145 between FN- and NR-fed BSFs affected bacterial community similarity at all stages of the BSF life cycle

146 (richness decay in Additional file 12) but did not influence community dispersion (i.e., within beta-

147 diversity: distance from the centroid in a multidimensional principal coordinate analysis (PCoA) space;

148 Additional file 13). The stressful conditions of NR diets shifted the configurations of gut bacterial

149 communities from a stable state to another [24]. During the juvenile stages (larvae and pupae), FN-fed

150 individuals had relatively stable bacterial communities that formed tight clusters in the ordination space

151 (Figures 2a and b); on the other hand, NR diets drove such communities toward new deterministic

152 configurations, resulting in distinct clusters in the ordination space with similar dispersion to FN (location

153 effects, [24]; Figures $2 \mathrm{~d}$ and e). On the contrary, in the adults where the morphogenetic events

154 affect/degenerate the internal tissues of insects [33], NR diets significantly affected the sample-to-sample

155 variability $\left(\mathrm{F}_{2,24}=18.3, p<0.0001\right)$, with destabilization of the bacterial communities and further

156 increment in microbiome stochasticity either increasing (NRF) or decreasing (NRV) microbiome

157 dispersion ([24]; Figures 2c and f).

158 The observed beta-diversity patterns and their related ecological drivers can be interpreted as the results

159 of the combination of two processes: species replacement (e.g., due to environmental filtering and 
160 competition) and richness differences (e.g., due to niche availability and physical barriers) [41]. In larvae,

161 pupae, and adults, within-diet beta-diversity was primarily driven by OTU replacement (average relative

162 contribution of $60 \%$ ), followed by richness differences (40\%; Figures $2 \mathrm{~g}-\mathrm{i}$ and Additional file 14); this

163 indicates that the different gut physicochemical conditions mediated by the diets in the midgut drive a

164 significant environmental filtering process (Additional file 5). This pattern was consistent across all BSF

165 developmental stages for all three diets, but with different magnitudes (larvae: $\mathrm{F}_{2,105}=14.5, p=0.001$;

166 pupae: $\mathrm{F}_{2,84}=8.9, p=0.001$; adult: $\mathrm{F}_{2,105}=6.6, p=0.001$; Figures $2 \mathrm{~g}-\mathrm{i}$ ), evidencing an overall higher

167 rate of replacement for the NRF diet (up to 74\%) than for the FN and NRV diets (up to 63\% and 58\%,

168 respectively; Additional file 14).

169

170 NR diets alter the bacterial community composition of BSF gut. As the NR diet-induced dysbiosis

171 progressed, the members of the BSF gut bacterial communities changed their composition and structure

172 (relative distribution in Figure $3 \mathrm{a}$ and Additional file 15), establishing diet-specific bacterial sub-

173 communities during BSF development (spheres in the ternary corners; Figure 3B). In the juvenile stages

174 (larvae and pupae), the main difference between the healthy (FN) and dysbiotic microbial communities

175 (NRF and NRV) was a drastic depletion in the main components of the bacterial community (Bacilli and

176 Gammaproteobacteria in larvae and pupae, respectively) compared with other community members. For

177 instance, in BSF larvae, the class of Bacilli-dominant in FN-fed individuals $(93 \%, 9 \%$, and $1 \%$ in FN,

178 NRV, and NRF, respectively) — was replaced with a combination of Gammaproteobacteria

179 (Enterobacteriaceae), Bacteroidia (Porphyromonadaceae), and Clostridia (Lachnospiraceae,

180 Ruminococcaceae, and Family XI) in NRF- and NRV-fed individuals (Figure 3a and Additional files 16

181 and 17). In the pupal stage, Clostridia bloomed in NR-fed individuals, suppressing the dominant

182 Gammaproteobacteria (Enterobacteriaceae) typical in FN-fed pupae (Figure 3a). In adults,

183 Gammaproteobacteria prevailed in all three diets $(52 \%, 64 \%$, and $92 \%$ in FN, NRF, and NRV, 
184 respectively), along with the presence of Bacilli in FN-fed individuals (34\%, 14\%, and 2\%) and

185 Alphaproteobacteria (Acetobacterales, 17.7\%, 6.4\%, and <1\%), Clostridia ( $<1 \%, 5 \%$, and 4\%), and

186 Alphaproteobacteria (Sphingomonadales, $<0.01 \%, 6.5 \%$, and 1.3\%) in NR-fed individuals (Figure 3a).

187 Notably, we detected a correlation between the relative abundance of the main bacterial classes and diet

188 components and BSF growth performance (Additional file 18). For example, the relative abundance of

189 Bacilli in larvae (dominating normobiotic microbiome) negatively correlated with moisture and

190 positively correlated with other diet components (carbohydrates, proteins, lipids, fiber, and others) and

191 larval weight. On the other hand, the abundance of Gammaproteobacteria and Clostridia (mainly

192 detected in dysbiotic microbiomes) had an opposite trend. In pupae, while Clostridia had similar patterns

193 of correlation with diet components, Gammaproteobacteria - dominant in FN-fed individuals—showed

194 a negative correlation with moisture and a positive correlation with all other diet components and pupae

195 weight (Additional file 18). Furthermore, in adults, Bacilli, indicators of a normobiotic condition, had a

196 positive correlation with host fitness (weight) and an FN regime (for all diet components), whereas

197 Clostridia and Gammaproteobacteria had negative correlations (Additional file 18).

198 The absence of a prevalent generalist community in favor of diet-specific bacterial sub-communities

199 (Figure 3b) and the strong correlation between diet components and growth and bacterial community

200 composition (Additional file 18) confirmed that the bacteria in the BSF gut were strongly selected by

201 deterministic processes driven by both NR and the developmental stages.

202

203 NR diets result in modified bacterial interactions in the microbial community of BSF gut. To assess

204 the biotic interactions among members of normobiotic (FN) and dysbiotic (NRs) bacterial communities

205 in BSF gut, we performed co-occurrence network analyses. We separately investigated highly significant

206 interactions between bacterial OTUs for each developmental stage (larvae, pupae, and adults) and diet, 
207 while also considering all the developmental stages together so as to detect conserved interactions 208 induced by diet type along the overall life cycle of the insect [42].

209 Despite the observed variability in the number of nodes and interactions among the developmental stages 210 and feeding diets (developmental stages in Additional file 19 and entire life in Table 2), in both FN- and

211 NR-fed individuals, mutualistic (positive) interactions were consistently higher than antagonistic

212 (negative) interactions, possibly due to their similar environmental preference and/or the high resistance

213 of gut microorganisms (commensals and/or symbiotic) to physiological gut modifications induced by the

214 diet (Additional file 5). However, comparison of the different diets revealed that antagonistic interactions

215 were enhanced in FN-fed insects (Table 2), suggesting the higher ability of FN bacterial assemblages to

216 counteract possible pathogens or opportunistic microorganisms [43,44].

217 Notably, the NR gut bacterial interactomes were consistently more rarefied (lower centralization and 218 higher modularity), less structured, and less connected (lower heterogeneity and density) than the FN gut 219 bacterial interactomes across all developmental stages (including the entire life span of insect; Figures 220 4a-d, Table 2, and Additional file 19). Due to this consistent disaggregation patterns, the co-occurrence 221 networks obtained from the analysis during the entire life span of the insects were considered to 222 determine the main taxa involved in the structuring of the interactomes under the three dietary regimens. 223 As expected, the composition of the FN and NR interactome nodes differed, partially reflecting the 224 bacterial taxonomic diversity described for the entire community (Additional file 20). Regardless of their 225 abundance, the most influential OTUs within the network were those with higher connections (i.e., hubs 226 and keystones). In particular, these OTUs - involved in community assembly, stability, and functionality 227 [45] — varied according to diet administration (hubs in Additional file 20 and keystones in Figure 4e). 228 For instance, FN-fed individuals showed five keystone OTUs all belonging to Bacilli (i.e., Leuconostoc, 229 Lactobacillus, Bacillus, and Weissella genera), while NR-fed individuals had a high number of highly 230 connected OTUs (8 and 10 in NRF and NRV, respectively) and included members of all their main 
231 bacterial groups, including Clostridia (Family XI) and Gammaproteobacteria (Acinetobacter,

232 Beggiatoaceae, and Burkholderiaceae) as well members of less abundant groups, including

233 Alphaproteobacteria (Devosia, Ochrobactrum, Kaistia, and Sphingomonas), Bacteroidia

234 (Dysgonomonas and Myroides) and "others" (Leucobacter and Flaviflexus genera of Actinobacteria; 235 Figure 4e).

236

237 Discussion

238

239 The debate on the significance of dysbiosis and its importance in affecting the growth, health, and

240 wellbeing of animal hosts is based on the hypothesis of a causal relationship between microbiome 241 configuration(s) in healthy individuals and their positive performance. However, such a causal 242 relationship is hard to demonstrate due to the circularity of the question [7]: What comes first - poor host 243 performance and unhealthy state or the associated microbiome configuration? In other words, is dysbiosis 244 a cause or a consequence of an unhealthy condition? In our study, we faced the same issue confounding 245 most other studies on dysbiotic microbiomes. We imposed stressful diet conditions on the BSF (i.e., NR) 246 and then compared these conditions with the conditions in BSF fed FN diet that well supports growth 247 and development. We chose NR diets as a stressor for inducing dysbiosis because they strongly perturb 248 the host and its microbiome [46] without introducing external direct effectors on and selectors of 249 microbiomes, such as toxic chemical compounds or virulence factors produced by microbial pathogens 250 or parasites. Our assumption was that by stressing the host/microbiome holobiont with NR regimens, we 251 could modify the gut microbiome $[47,48]$ to "detrimental configurations," which would weaken host 252 resilience [2,9] - a requisite for defining the change in microbiome configuration as dysbiotic [24]. In 253 our study, we used two unrelated NR diets that may result in NR or caloric restriction stresses to the BSF.

254 The FN diet was optimal in sustaining insect growth and development along the entire life cycle; this 
allowed us to obtain heavier insects in a shorter time, likely due to the higher carbohydrate, lipid, and 256 protein contents in the FN diet than in the NR diets [31,34,49,50]. The good growth performance of the 257 host suggests that the gut microbiome configuration under the FN diet is a normobiotic configuration, 258 leading to the circular definition considered above; therefore, the microbiome configurations identified under the NR conditions are dysbiotic (i.e., refer to BSF growth performance and gut physicochemical 260 conditions; Figure 1 and Additional file 5; [36,37,40,51]). This problem in interpreting the role and 261 competence of a microbiome configuration is due to the lack of a univocal rather than comparative 262 definition of dysbiosis [15]. However, the general variability in selection conditions, including host 263 genotype, variable diets, and different environmental conditions under which the host lives $[22,46,52-$ 55], suggests that there can be multiple normobiotic and dysbiotic microbiome configurations, with a full causative effect on host performance and health [11,24].

266 The multiple configurations of normobiotic and dysbiotic microbiomes can be subjected to changes 267 driven by selection energy inputs that favor the passage from one configuration to another due to 268 changing and stronger selective forces [1,7,24]. In this context, it has been proposed under the frame of the Anna Karenina Principle (AKP) [56] that stressful conditions and the related microbial configurations represent the stochastic perturbations that can induce microbiome destabilization, thereby resulting in more dispersed microbial communities in affected individuals [24]. It has been postulated that severe stress around a healthy "core microbiome" can expand the dispersion of the microbial configurations with large halos or smears of possible microbiomes. In NR-fed BSF juveniles (larvae and pupae), rather than observing such increments in dispersion (i.e., within beta-diversity: distance from the centroid in the ordination space; Figures $2 \mathrm{~d}$ and f), we observed alternative stable states of the microbiomes driven by diet (i.e., distinct clusters in the ordination space: location effects) [24]. Contrastingly, in adults, the microbiomes were altered in unpredictable ways (i.e., with different dispersions). However, adults have 278 a particular gut system which undergoes morphogenetic events, which in turn affect the internal tissues; 
279 this possibly posing some limitations on gut functionality [33]. The location effect-driven by diet 280 (deterministic change) rather than by the increment in dispersion (stochastic change) in NR-fed juvenile 281 microbiome configurations - can be explained by the robustness of the immune system of the BSF, which 282 may act as a barrier preventing random modification of the bacterial community under changing 283 conditions. A study recently showed that BSF larvae can produce a remarkably expanded spectrum of 284 antimicrobial peptides (AMPs), many of which are expressed in response to changing nutritional 285 conditions or high bacterial loads [57]. The authors hypothesized that this expanded spectrum and diet286 dependent expression of AMPs is essential for BSF larvae to adapt to nutritionally unpredictable 287 substrates that are often highly contaminated with potential pathogens. However, such a consideration 288 cannot be extended to adults because in their case, the microbiome assembly is more dynamic [23] and 289 possibly driven by morphogenetic events (i.e., BSF larval midgut is completely removed during 290 metamorphosis) [33] and undefined stochastic factors.

291 Because the two NR diets strongly affected BSF growth and development and selected different 292 microbiome configurations without affecting the range of dispersion, we conclude that within beta293 diversity cannot be considered a key indicator of the unhealthy state of the BSF under NR regimens. The 294 results point out that under an NR stressor, BSF juvenile guts do not follow the AKP effects discussed 295 by Zaneveld et al. [24] but establish new stable and alternative "stressed" bacterial community configurations driven by deterministic changes induced by NR.

According to our initial hypothesis, NR diets did not affect bacterial community dispersion but the bacterial interactome in BSF gut, and in particular, the centralization parameters of the network. A denser, more structured, and more connected bacterial interactome was selected by the FN diet, a condition associated with efficiently performing microbiomes [58], including the counteraction of 301 possible pathogens or opportunistic microorganisms [43,44], stabilization of microbial configurations, 302 and promotion of network stability and gut homeostasis [59]. In contrast, networking of NR-fed 
303 individuals resulted in less structured configurations, with separate loosely interconnected node hubs-

304 a condition more typical during stresses $[60,61]$ that can negatively impact both the host and the function

305 of the microbiome. In FN-fed individuals, Bacilli belonging to the genera Leuconostoc, Lactobacillus,

306 Weissella, and Bacillus were denoted as the keystone species of the interactome. The first three of these

307 genera are lactic acid bacteria and are known as favorable microorganism due to their ability to stimulate

308 host gastrointestinal development and digestive function (carbohydrate, peptide, and lipid metabolism)

309 [22,62], immune response, and improved disease resistance [63]. Administration to the BSF of Bacilli

310 isolated from BSF larval gut, such as Bacillus subtilis, has reportedly shortened the development period

311 of BSF from larval to adult stage and has significantly increased its growth when reared on poultry

312 manure [64]. This highlights the importance of a stable diet-specific bacterial assemblage, in which the

313 assembled Bacilli are primarily involved $[46,62,65]$ in sustaining BSF growth and development. Instead,

314 in both the NR interactomes, Bacteroidia, Gammaproteobacteria, Alphaproteobacteria, and Clostridia,

315 along with less abundant groups, were the main keystone taxa. These taxa have been previously found

316 as dominant members in BSF fed with food waste and poultry manure [22] and have also been proposed

317 as potential microbial signatures of diseases [65].

318

319 Conclusions

320

321 The saprophagous and omnivorous BSF represents a versatile model to explore the effects of different

322 diets on the configuration and interaction of the host microbiome. The present study shows how the diet

323 source is primarily involved in shaping the bacterial microbiome composition and networks in the gut of

324 BSF, rather than the microbial community dispersion. NR determines changes of the gut bacterial

325 community to new stable non-dispersed configurations which are dysbiotic because do not support the

326 host resilience. These communities present a highly disintegrated network in which key components that 
327 sustain network connections and host homeostasis (e.g., bacilli) are substituted by other bacteria

328 incapable of sustaining the resilience of the gut ecosystem and the holobiont. We conclude that it is

329 important to understand the variation and network properties of the microbiome associated with dysbiosis

330 and their correlation with host performance to define the strategies to sustain insect growth on unbalanced

331 diets (i.e., organic waste).

333 Methods

335 Diet composition and insect growth. BSF specimens were reared at the entomological facilities of the 336 University of Milan using the methods described by Jucker et al. [31]. Three different feeding diet regimens were used: a FN diet, comprising 50\% wheat germ, 30\% alfalfa, and 20\% corn flour, to which an equal volume of water was added; a NRF diet, comprising apples, pears and, oranges, each at $33.3 \%$; and a NRV diet, comprising green bean, cabbage, and lettuce, each at 33\%. The chemical composition 340 of the FN diet was obtained from the diet's ingredients provider (Laboratorio Dottori Piccioni s.r.l.,

341 Gessate, Milano), whereas that of NRF and NRV was extrapolated from the food composition database

342 (http://nut.entecra.it; Additional file 1). The two NR diets were selected because they had (i) lower nutrient contents (proteins, lipids, carbohydratess and fiber) than those supplied by the control FN diet and (ii) different carbohydrate-to-protein ratio (an important diet parameter for BSF development [66]; Additional file 1). In addition, NR diets were prepared using different ingredients from the FN diet (i.e., fruits and vegetables) to exert a different selective pressure $[46,67]$ and mimic the variability of the natural diets to which the BSF could be exposed to (e.g., horticulture waste) $[31,67,68]$.

348 From the stock culture of a laboratory fly, the eggs of H. illucens were collected on cardboard strips for 349 oviposition and then transferred into plastic containers $(10.5 \times 5 \mathrm{~cm})$ with metal webs to allow air exchange and containing the three diets (FN, NRF, and NRV). These containers were kept in a climate 
351 chamber under controlled conditions (temperature: $25^{\circ} \mathrm{C} \pm 0.5^{\circ} \mathrm{C}$; relative humidity: $60 \% \pm 5 \%$;

352 photoperiod: 12:12 light:dark). The handling larvae $(\mathrm{n}=400$ per diet $)$ were provided ad libitum access

353 to the three diets. Emerging pupae were transferred into three different cages according to the feeding

354 diet and without a new food source until the eclosion of adults. The following data were recorded: larval

355 (measured after egg eclosion, when $40 \%$ of larvae reached the pupal stage), pupae, and adult weight

356 (Sartorius CP64 analytical balance, Germany); pupae and adult length (mm); and the survival of 357 individuals in each developmental stage. Fresh weights were determined for larvae and pupae, whereas 358 dry weight was determined for adults after desiccation at $105^{\circ} \mathrm{C}$ for $48 \mathrm{~h}$. For larvae, growth rate was 359 also calculated and expressed as average fresh weight (g) per day of growth. Correlation (Pearson, 360 Kendall, and Spearman, $p<0.05$ ) was assessed using the $\mathrm{R}$ software to measure the strength of the 361 association between growth performance (i.e., weight) and diet components.

362 In addition to insect growth performance analysis, physicochemical conditions (oxygen partial pressure, $363 \mathrm{pH}$, and redox potential) were also measured using the methods described in Additional file 21. For these 364 measurements, only fourth instar larvae were used because gut functionality is not affected by a non365 continuous or stopped feeding behavior nor by the remodeling processes that are associated with the 366 following developmental stages (i.e., pupae and adult) [33]. Analysis of variance (ANOVA) was 367 performed to evaluate the differences in BSF growth performance and physicochemical conditions 368 among the three diets.

370 Sampling, sterilization, and dissection of insects. BSF individuals fed on the three diets (FN, NRF, 371 and NRV) were sampled at different developmental stages (Additional file 3): larvae, pupae (indicated 372 by the change in their color from creamy-white to black), and adults (both female and male, immediately 373 after eclosion). The surface was sterilized prior to insect dissection [69]. Briefly, after a first wash with $3740.1 \%$ sodium dodecyl sulfate in $50-\mathrm{ml}$ tubes, BSF individuals were immersed in $1 \%$ sodium hypochlorite 
375 for $10 \mathrm{~min}$, followed by three consecutive washes with $70 \%$ ethanol and five washes with sterilized

376 distilled water. The gastrointestinal tract was dissected from larvae and pupae under sterile conditions.

377 For adults, because their digestive tracts were fragile due to the non-feeding status of the emerged

378 individuals, we used the entire body deprived of the head, legs, and wings. For cultivation-independent 379 analysis, a total of 81 samples (9 replicates per each diet at each developmental stage) were stored in 380 single 1.5 -ml tube containing $98 \%$ ethanol at $-20^{\circ} \mathrm{C}$.

382 DNA extraction from insects. Metagenomic DNA was extracted from BSF individuals using the

383 DNeasy Blood \& Tissue Kit (Qiagen, Milano, Italy). The samples stored at $-20^{\circ} \mathrm{C}$ were centrifuged for

$3845 \mathrm{~min}$ at $3000 \mathrm{rpm}$. After ethanol removal, the tissues were hydrated by adding $1 \mathrm{ml}$ of sterile 385 physiological solution $(0.9 \% \mathrm{NaCl})$. Then, the samples were centrifuged for $5 \mathrm{~min}$ at $3000 \mathrm{rpm}$. The 386 solution was removed and $180 \mu 1$ of ATL buffer (provided by the kit) was added. Tissues were 387 homogenized in ATL buffer. Alternate incubations at $-80{ }^{\circ} \mathrm{C}$ and $70{ }^{\circ} \mathrm{C}$ for 10 minutes each were 388 performed. Then, $25 \mu 1$ of lysozyme $(20 \mathrm{mg} / \mathrm{ml})$ was added to the homogenate and the samples were 389 incubated at $37^{\circ} \mathrm{C}$ for 30 min to digest the gram-positive walls so as to release the bacterial DNA. The 390 final steps were performed following the manufacturer's instructions. The quality of the extracted DNA 391 was checked using the Nanodrop 1000 spectrophotometer (BioTek $^{\circledR}$, PowerWave XS2).

393 Fingerprinting and high-throughput sequencing of the bacterial communities. The variations in the 394 bacterial communities of insects exposed to different diets in each developmental stage were investigated 395 using PCR fingerprinting (ARISA) [70] and high-throughput sequencing (Illumina) of the ITS and 16S 396 rRNA gene, respectively. For ARISA-PCR fingerprinting, the ITS-F FAM (5'-GTC GTA ACA AGG 397 TAG CCG TA-3') and ITS-R (5'-GCC AAG GCA TCC ACC-3') primer pairs were used, following the 398 protocol described in Additional file 22. The extracted DNA was further sequenced at King Abdullah 
399 University of Science and Technology (Bioscience Core Lab) using the primers 341f and 785r (the V3-

400 V4 hypervariable region of the 16S rRNA gene) [71] and following the protocol described by Mapelli et

401 al. [72]. The raw data obtained from Illumina sequencing were analyzed using a combination of the

402 Quantitative Insights into Microbial Ecology (QIIME) pipeline version 1.8 [73] and UPARSE version 8

403 [74], as described by Marasco et al. [43]. Representative sequences of each OTU97 were aligned in

404 QIIME using UClust and searched against the SILVA 128 database for bacteria. Captured diversity was

405 visualized using computing rarefaction curves for each sample (Additional file 23). Sequence reads were

406 deposited in the NCBI SRA database under SRA accession PRJNA421313.

407 Bray-Curtis dissimilarity distance matrices were obtained from the ARISA-ITS quantitative and the log408 transformed-quantitative OTU matrices. Both BC matrices were used to perform PCoA [75] for each 409 developmental stage (larvae, pupae, and adult [female and male]); no differences in the bacterial 410 communities associated with female and male adults developed within the same diet were observed 411 (Additional file 7); therefore, they were considered as belonging to the same group (i.e., the adult group) 412 in our experimental design and further analyses. A multivariate generalized linear model (manyglm) [76] 413 and multivariate linear model (manylm) [77] were used for the 16S rRNA gene and ITS datasets, 414 respectively, considering diet as the fixed and orthogonal explanatory variable (three levels: FN, NRF, 415 and NRV diets) for each developmental stage.

416 Using the 16S rRNA gene dataset, the variance was partitioned using the varpart function within the 417 Vegan package in R [78]. Decay analysis was performed to evaluate the decrease in growth performance 418 (i.e., weight) in larvae, pupae, and adults fed with the three diets with respect to the function of the gut 419 bacterial community similarity. The cumulative proportion of bacterial community variation $\left(\mathrm{R}^{2}\right)$, as 420 explained by diet components (predictor variables), was calculated using the sequential test in addition 421 to the corrected Akaike information criterion [79] within distance-based multivariate analysis for a linear 422 model [80]. The components of beta-diversity (similarity, replacement, and difference in richness) were 
423 calculated using the beta.div.comp function of the R package adespatial v0.3-8 [41]. Alpha diversity

424 indices were calculated using the Paleontological Statistics Software Package for education and data 425 analysis [81]. ANOVA (Tukey's multiple comparison tests) was performed to test differences in alpha 426 diversity indices among the different diets across the three developmental stages using the GraphPad 427 software. The relative abundance and distribution of the OTUs were visualized with ternary plots using 428 the ggtern software package in R [82]. The Non-parametric Kruskal-Wallis (false discovery rate [FDR], 429 $p$-correction) and Dunn's multiple comparisons tests were used to detect the differences in the taxonomic groups (class level) in the three diets across the developmental stages using GraphPad. The correlation 431 between the taxonomical composition of the bacterial communities (class level) and diet components were determined using the combination of three methods (Pearson, Kendall, and Spearman) in R.

434 Co-occurrence network analysis. To explore the significant relations among the OTUs, a non-random 435 structure of co-occurrence network was constructed for each bacterial community inhabiting the 436 digestive tract of BSFs fed with the three diets (FN, NRF, and NRV) [43] by considering (i) each 437 developmental stage separately (larvae, pupae, and adult) and (ii) the overall life span of the insect 438 (merging of the three developmental stages). The routine CoNet in Cytoscape $3.4[29,83]$ was applied 439 using the computational methods described by Marasco et al. [84]. To identify the strongest significant 440 interactions among the OTUs with relative abundances of $>0.01 \%$, two correlation (Spearman and 441 Pearson, $p$-value) and two distance-based (Bray-Curtis and Kullback-Leibler, $z$-value methods were 442 used and their results were merged using the Fisher's method and corrected for multiple tests (Benjamini443 Hochberg correction). The network null distribution was computed and used to detect the significant 444 interactions; edges (i.e., interactions between two nodes) with $p$-values of $<0.01$ were discarded [83]. 445 The topological indices of the networks were calculated using Cytoscape [85]. In the case of the overall 446 life co-occurrence networks, visualization was performed using Gephi [86]; statistical differences 
447 between the degrees of connection in the co-occurrence networks for the different diets were analyzed 448 via ANOVA using R prior to performing the normality and homoscedasticity tests $[87,88]$. Nodes with 449 a connection frequency of $>75 \%$ were also identified as network hubs, whereas the hub-nodes with the 450 highest level of degree of connection and betweenness centrality were considered keystone species [45].

452 List of abbreviations. BSF: black soldier fly; NR: nutrient restriction; FN: full nutrient; NRF: nutrient 453 restriction fruit; NRV: nutrient restriction vegetable; WRI: waste reduction index; ECI: efficiency of 454 conversion of ingested food; $\mathrm{pH}$ : power of hydrogen; AMPs: antimicrobial peptides; ITS: internal 455 transcribed spacers; PCoA: principal coordinate analysis; OTU: operational taxonomic unit; AKP: Anna 456 Karenina principle; ANOVA: analysis of variance; DNA: deoxyribonucleic acid; PCR: polymerase chain reaction; ARISA: automated ribosomal intergenic spacer; QIIME: quantitative insights into microbial ecology; NCBI: national center for biotechnology information; SRA: sequence read archive; manyglm: multivariate generalized linear model; manylm: multivariate linear model; BC: Bray-Curtis.

Ethics approval and consent to participate. Not applicable

462 Consent for publication. Not applicable

463 Availability of data and material. The dataset supporting the conclusions of this article is available in 464 the NCBI SRA database [BioProject PRJNA421313].

465 Competing interests. The authors declare that they have no competing interests.

466 Funding. King Abdullah University of Science and Technology supported the study through baseline 467 research funds to DD. EC acknowledges personal supports from "Piano Sviluppo di Ateneo: Linea B468 Dotazione annuale per attività istituzionale" in the project "Insects to feed the future: a new sustainable 469 protein source (INSPIRE)" and "Piano di Sostegno della Ricerca 2015-2017: Linea 2 - Dotazione annuale 470 per attività istituzionali" in the project "Microbial interactions in complex ecosystems (MIRACLE)." 
471 Authors' contributions. RM, MF, EC, and DD conceived and designed the experiments. MC, CJ, and 472 SS collected the samples. MC, RM, MF, JC, SS, and EC performed the experiments. RM, MF, CJ, SS, 473 and FM analyzed the data. DD, SB, and EC contributed reagents/materials/analysis tools. RM, MF, DD, 474 and EC wrote the paper: All authors critically revised the manuscript.

475 Acknowledgments. King Abdullah University of Science and Technology supported the study through 476 baseline research funds to DD. EC acknowledges personal supports from "Piano Sviluppo di Ateneo: 477 Linea B-Dotazione annuale per attività istituzionale" in the project "Insects to feed the future: a new 478 sustainable protein source (INSPIRE)" and "Piano di Sostegno della Ricerca 2015-2017: Linea 2 479 Dotazione annuale per attività istituzionali" in the project "Microbial interactions in complex ecosystems 480 (MIRACLE).”

481

482 Supplementary information

483 Additional file 1: Table S1. Chemical composition of rearing substrates: FN, full nutrient; NRF, nutrient 484 restriction fruit; NRV, nutrient restriction vegetable. Values in the brackets indicate reduction (-) or 485 increase $( \pm)$ of nutrients' fresh weight expressed as percentage respect to $\mathrm{FN}$ as standard $(100 \%)$.

486 Additional file 2: Table S2. Length of pupae and adult individuals. Lower case letters indicate the results 487 of Tukey's multiple comparison tests. FN, full nutrient; NRF, nutrient restriction fruit; NRV, nutrient 488 restriction vegetable.

489 Additional file 3: Figure S1. Representative images of BFS larvae, pupae (black color) and adults fed 490 on FN, NRF and NRV diets. Bar scale indicate $10 \mathrm{~mm}$.

491 Additional file 4: Table S3. Correlation among the different components of the three diets (FN, NRF 492 and NRV) and the weight of individuals at each developmental stage. Pearson, Kendall and Spearman 493 correlation, $p<0.05$. 
494 Additional file 5: Figure S2. Microprofiles of (a and b) oxygen partial pressure $(\mu \mathrm{m} / \mathrm{L})$, (c and d) $\mathrm{pH}$ 495 and (e and f) redox potential $(\mathrm{mV})$ along the gut compartments of larvae reared on full nutrient (FN, 496 black), nutrient restriction fruit (NRF, orange) and nutrient restriction vegetable (NRV, green) diets. (a, $497 \mathrm{c}$ and e) Values are given as average \pm standard error $(\mathrm{n}=7)$. (g) Image of BSF gut. Scale bar $1 \mathrm{~cm} .(\mathrm{b}, \mathrm{d}$ 498 and f) Representative radial profiles of oxygen partial pressure, $\mathrm{pH}$ and redox potential, respectively, in the midgut of BSF fed on the three diets. Depth $(\mu \mathrm{m})$ refers to the sensor tip position along the midgut. 500 Under the radial profiles, a schematic representation of oxygen (light blue spheres), $\mathrm{pH}$ (yellow spheres) 501 and redox (gray spheres) gradients showing the effect of the three diets on the physiological status of the midgut.

Additional file 6: Figure S3. PCoA-analysis based on ARISA Bray-Curtis dissimilarities matrices of gut' bacterial communities associated with (a) larvae, (b) pupae and (c) adult fed on FN (full nutrient, 505 black), NRF (nutrient restriction fruit, orange), and NRV (nutrient restriction vegetable, green).

Additional file 7: Table S4. Multivariate GLM analysis applied on the (a) 16S rRNA gene and (b) 16S23S rRNA internal transcribed spacers (ITS) datasets. Difference among bacterial community associated with female and male are also assessed for each diet to ensure that all adult* can be analyzed as a unique developmental stage. Results of pairwise comparison (t-test) are reported for (c) 16S rRNA gene and (D) 16S-23S rRNA internal transcribed spacers (ITS) adult' datasets, respectively.

Additional file 8: Table S5. Variation partitioning analysis of bacterial community explained by diets (FN, NRF and NRV) at each developmental stage was assessed using vegan package in R.

Additional file 9: Table S6. Results of DistLM sequential tests of diet components (Additional file 1: Table S1) at each developmental stage. Cumulative proportion of variation $\left(\mathrm{R}^{2}\right)$ in bacterial community structure explained by fitting variables (i.e., diet components) within sets sequentially using forward 516 selection, and conditional tests using 999 permutations of residuals. Diet components included in the 517 sequential test are reported, along with AICc, Pseudo-F, $p$-value and cumulative $\mathrm{R}^{2}$. 
518 Additional file 10: Figure S4. Relationship between bacterial communities' similarities (Bray-Curtis) 519 and the weight decay of (a) larvae, (b) pupae, and (c) adult fed on NR and NR diets. R-squared values of 520 regression line and statistical significance of correlation among the two variables $(p<0.05)$ are shown in 521 the graph.

Additional file 11: Table S7. Diversity indices describing the bacterial community associated to BSF subjected to different diet regimes along the development stages (i.e., larvae, pupae and adult). Richness indicates the total number of OTUs for each sample; Evenness is represented by Pielou's index and quantifies how equal the community is numerically. All the values are reported as average \pm standard deviation; number of replicates indicated as (n). Lower case letters indicate the results of multiple comparison tests for each developmental stage. FN, full nutrient; NRF, nutrient restriction fruit; NRV, nutrient restriction vegetable.

Additional file 12: Figure S5. Relationship between bacterial communities' similarities (Bray-Curtis) and the richness decay of (a) larvae, (b) pupae, and (c) adult fed on the different diets (FN, NRF and NRV). R-squared values of regression line and statistical significance of correlation among the two variables $(p<0.05)$ are shown in the graph.

Additional file 13: Figure S6. Relationship between within beta-diversity (i.e., dispersion) and the richness (number of OTUs) of gut bacterial communities associated with (a) larvae, (b) pupae, and (c) adult fed on the different diets (FN, NRF and NRV). R-squared values of regression line and statistical significance of correlation among the two variables $(p<0.05)$ are shown in the graph.

Additional file 14: Table S8. Within beta-diversity components (species replacement and differences in OTUs richness) expressed as average \pm standard deviations of the pairwise similarities of each diet (FN, NRF and NRV) along the developmental stages. Ratio among replacement and richness difference processes is also reported. Star $\left(^{*}\right)$ indicate the case in which replacement not dominate on richness 
542 brackets. Lower case letters indicate the results of the Tukey's multiple comparison test for replacement

543 (upper panels) and richness difference (lower panels) at each developmental stage.

544 Additional file 15: Figure S7. Het map of bacterial classes' relative abundance (\%) in larvae, pupae and 545 adults fed on FN and NR diets.

546 Additional file 16: Table S9. Taxonomy detailed tables. (a) Class, (b) order and (c) family levels. See

547 Marasco et al 2020_Additional file 16_Table S9.xlsx

548 Additional file 17: Figure S8. Relative abundance of the main bacterial classes detected in BFS fed with

549 FN and NR diets. Lower case letters indicate the results of the Dunn's multiple comparison test among 550 diets (FN, NRF and NRV) at each developmental stage.

551 Additional file 18: Table S10. Table resuming correlation of the different components of the three diets

552 (FN, NRF and NRV) and growth performance (weight) with the main taxa (class level) detected in larvae, 553 pupae and adults. Values indicated the correlation coefficient and stars $\left(^{*}\right)$ the results of Pearson, Kendall 554 and Spearman correlation tests $\left(*, p<0.05 ;{ }^{* *}, p<0.01 ; * * *, p<0.001\right)$.

555 Additional file 19: Table S11. Co-occurrence network topology indices reported for larvae (L), pupae 556 (P) and adults (A) fed on FN, NRF and NRV diets. Star $\left(^{*}\right)$ indicate the indices expressed as percentage 557 of increment (+) or decrement (-) respect to the FN.

558 Additional file 20: Figure S9. Relative abundance of the main bacterial classes detected in BFS fed with

559 FN and NR diets. Results are reported for the (a) total bacterial communities (total number of reads), (b) 560 networks' nodes ( $\mathrm{n}$ indicate the total number of network nodes) and (c) networks' hubs ( $\mathrm{n}$ indicate the 561 total number of network hubs).

562 Additional file 21: Methods S1. Measurement of physicochemical conditions of BFS gut comportments.

563 Additional file 22: Methods S2. ARISA fingerprinting analysis of bacterial communities. 
564 Additional file 23: Figure S10. Rarefaction curve of 16S rRNA gene sequencing of (a) larvae, (b) pupae

565 and (c) adult consuming three different diets (FN, full nutrient=black; NRF, nutrient restriction 566 fruit=orange; NRV, nutrient restriction vegetable=green).

\section{References}

1. Levy M, Kolodziejczyk AA, Thaiss CA, Elinav E. Dysbiosis and the immune system. Nat Rev 571 Immunol 2017;17:219-32.

572 2. Sommer F, Anderson JM, Bharti R, Raes J, Rosenstiel P. The resilience of the intestinal microbiota 573 influences health and disease. Nat Rev Microbiol 2017;15:630-8.

574 3. Yatsunenko T, Rey FE, Manary MJ, Trehan I, Dominguez-Bello MG, Contreras M, et al. Human gut 575 microbiome viewed across age and geography. Nature 2012;486:222-7.

576 4. Montagna M, Chouaia B, Mazza G, Prosdocimi EM, Crotti E, Mereghetti V, et al. Effects of the diet 577 on the microbiota of the red palm weevil (Coleoptera: Dryophthoridae). PLoS One 2015;10:e0117439.

578 5. Wu GD, Chen J, Hoffmann C, Bittinger K, Chen Y-Y, Keilbaugh SA, et al. Linking long-term dietary 579 patterns with gut microbial enterotypes. Science 2011;334:105-8.

580 6. Vacchini V, Gonella E, Crotti E, Prosdocimi EM, Mazzetto F, Chouaia B, et al. Bacterial diversity 581 shift determined by different diets in the gut of the spotted wing fly Drosophila suzukii is primarily 582 reflected on acetic acid bacteria. Environ Microbiol Rep 2017;9:91-103.

583 7. Hooks KB, O’Malley MA. Dysbiosis and its discontents. MBio. 2017;8:1-11.

584 8. Hamdi C, Balloi A, Essanaa J, Crotti E, Gonella E, Raddadi N, et al. Gut microbiome dysbiosis and 585 honeybee health. J Appl Entomol 2011;135:524-33.

586 9. Lozupone CA, Stombaugh JI, Gordon JI, Jansson JK, Knight R. Diversity, stability and resilience of 587 the human gut microbiota. Nature 2012;489:220-30. 
10. Clark RI, Salazar A, Yamada R, Fitz-Gibbon S, Morselli M, Alcaraz J, et al. Distinct shifts in 589 microbiota composition during drosophila aging impair intestinal function and drive mortality. Cell Rep $590 \quad 2015 ; 12: 1656-67$.

591 11. Ma Z. Testing the Anna Karenina principle in human microbiome-associated diseases. iScience $592 \quad 2020 ; 23$.

593 12. David LA, Maurice CF, Carmody RN, Gootenberg DB, Button JE, Wolfe BE, et al. Diet rapidly and 594 reproducibly alters the human gut microbiome. Nature 2014;505:559-63.

595 13. Chaturvedi S, Rego A, Lucas LK, Gompert Z. Sources of variation in the gut microbial community 596 of Lycaeides melissa caterpillars. Sci Rep 2017;7:11335.

597 14. Cabana F, Clayton JB, Nekaris KAI, Wirdateti W, Knights D, Seedorf H. Nutrient-based diet 598 modifications impact on the gut microbiome of the Javan slow loris (Nycticebus javanicus). Sci Rep $599 \quad 2019 ; 9: 4078$.

600 15. Gentile CL, Weir TL. The gut microbiota at the intersection of diet and human health. Science $601 \quad 2018 ; 362: 776-80$.

602 16. Denou E, Marcinko K, Surette MG, Steinberg GR, Schertzer JD. High-intensity exercise training 603 increases the diversity and metabolic capacity of the mouse distal gut microbiota during diet-induced 604 obesity. Am J Physiol Metab 2016;310:E982-93.

605 17. Sonnenburg ED, Smits SA, Tikhonov M, Higginbottom SK, Wingreen NS, Sonnenburg JL. Diet606 induced extinctions in the gut microbiota compound over generations. Nature 2016;529:212-5.

607 18. Sannino DR, Dobson AJ, Edwards K, Angert ER, Buchon N. The Drosophila melanogaster gut 608 microbiota provisions thiamine to its host. MBio 2018;9:e00155-18.

609 19. Chouaia B, Goda N, Mazza G, Alali S, Florian F, Gionechetti F, et al. Developmental stages and gut 610 microenvironments influence gut microbiota dynamics in the invasive beetle Popillia japonica Newman 611 (Coleoptera: Scarabaeidae). Environ Microbiol 2019;21:4343-59. 
612 20. Kakumanu ML, Maritz JM, Carlton JM, Schal C. Overlapping community compositions of gut and 613 fecal microbiomes in lab-reared and field-collected german cockroaches. Appl Environ Microbiol $6142018 ; 84: 1-17$.

615 21. Tang Y, Lian B, Dong H, Liu D, Hou W. Endolithic bacterial communities in dolomite and limestone 616 rocks from the Nanjiang Canyon in Guizhou karst area (China). Geomicrobiol J 2012;29:213-25.

617 22. Jiang C, Jin W, Tao X, Zhang Q, Zhu J, Feng S, et al. Black soldier fly larvae (Hermetia illucens) 618 strengthen the metabolic function of food waste biodegradation by gut microbiome. Microb Biotechnol $6192019 ; 12: 528-43$.

620 23. Hammer TJ, Moran NA. Links between metamorphosis and symbiosis in holometabolous insects. 621 Philos Trans R Soc B Biol Sci. 2019;374:20190068.

622 24. Zaneveld JR, McMinds R, Vega Thurber R. Stress and stability: applying the Anna Karenina 623 principle to animal microbiomes. Nat Microbiol 2017;2:17121.

624 25. Józefiak D, Józefiak A, Kierończyk B, Rawski M, Świątkiewicz S, Długosz J, et al. 1. Insects - a 625 natural nutrient source for poultry - a review. Ann Anim Sci 2016;16:297-313.

626 26. Nguyen TTX, Tomberlin JK, Vanlaerhoven S. Ability of black soldier fly (Diptera: Stratiomyidae) 627 larvae to recycle food waste. Environ Entomol 2015;44:406-10.

628 27. De Marco M, Martínez S, Hernandez F, Madrid J, Gai F, Rotolo L, et al. Nutritional value of two 629 insect larval meals (Tenebrio molitor and Hermetia illucens) for broiler chickens: Apparent nutrient 630 digestibility, apparent ileal amino acid digestibility and apparent metabolizable energy. Anim Feed Sci 631 Technol 2015;209:211-8.

632 28. Kroeckel S, Harjes A-GE, Roth I, Katz H, Wuertz S, Susenbeth A, et al. When a turbot catches a fly: 633 Evaluation of a pre-pupae meal of the Black Soldier Fly (Hermetia illucens) as fish meal substitute 634 Growth performance and chitin degradation in juvenile turbot (Psetta maxima). Aquaculture 2012;364$635365: 345-52$. 
636 29. Faust K, Sathirapongsasuti JF, Izard J, Segata N, Gevers D, Raes J, et al. Microbial co-occurrence 637 relationships in the human microbiome. Ouzounis CA, editor. PLoS Comput Biol.2012;8:e1002606.

638 30. Liao C-Y, Rikke BA, Johnson TE, Diaz V, Nelson JF. Genetic variation in the murine lifespan 639 response to dietary restriction: from life extension to life shortening. Aging Cell 2010;9:92-5.

640 31. Jucker C, Erba D, Leonardi MG, Lupi D, Savoldelli S. Assessment of vegetable and fruit substrates 641 as potential rearing media for Hermetia illucens (Diptera: Stratiomyidae) larvae. Environ Entomol $642 \quad 2017 ; 46: 1415-23$.

643 32. Tettamanti G, Grimaldi A, Casartelli M, Ambrosetti E, Ponti B, Congiu T, et al. Programmed cell 644 death and stem cell differentiation are responsible for midgut replacement in Heliothis virescens during 645 prepupal instar. Cell Tissue Res 2007;330:345-59.

646 33. Bruno D, Bonelli M, Cadamuro AG, Reguzzoni M, Grimaldi A, Casartelli M, et al. The digestive 647 system of the adult Hermetia illucens (Diptera: Stratiomyidae): morphological features and functional 648 properties. Cell Tissue Res 2019;378:221-38.

649 34. Pimentel AC, Montali A, Bruno D, Tettamanti G. Metabolic adjustment of the larval fat body in 650 Hermetia illucens to dietary conditions. J Asia Pac Entomol 2017;20:1307-13.

651 35. Gold M, Tomberlin JK, Diener S, Zurbrügg C, Mathys A. Decomposition of biowaste macronutrients, 652 microbes, and chemicals in black soldier fly larval treatment: A review. Waste Manag 2018;82:302-18. 653 36. Valzania L, Coon KL, Vogel KJ, Brown MR, Strand MR. Hypoxia-induced transcription factor 654 signaling is essential for larval growth of the mosquito Aedes aegypti. Proc Natl Acad Sci USA $655 \quad 2018 ; 115: 201719063$.

656 37. Coon KL, Valzania L, McKinney DA, Vogel KJ, Brown MR, Strand MR. Bacteria-mediated hypoxia 657 functions as a signal for mosquito development. Proc Natl Acad Sci USA 2017;114:E5362-9.

658 38. Kim W, Bae S, Park K, Lee S, Choi Y, Han S, et al. Biochemical characterization of digestive 659 enzymes in the black soldier fly, Hermetia illucens (Diptera: Stratiomyidae). J Asia Pac Entomol 
661 39. Šustr V, Stingl U, Brune A. Microprofiles of oxygen, redox potential, and $\mathrm{pH}$, and microbial 662 fermentation products in the highly alkaline gut of the saprophagous larva of Penthetria holosericea 663 (Diptera: Bibionidae). J Insect Physiol 2014;67:64-9.

664 40. Reese AT, Cho EH, Klitzman B, Nichols SP, Wisniewski NA, Villa MM, et al. Antibiotic-induced 665 changes in the microbiota disrupt redox dynamics in the gut. Elife 2018;7:1-22.

666 41. Legendre P. Interpreting the replacement and richness difference components of beta diversity. Glob 667 Ecol Biogeogr 2014;23:1324-34.

668 42. Faust K, Raes J. Microbial interactions: from networks to models. Nat Rev Microbiol 2012;10:53866950.

670 43. Marasco R, Rolli E, Fusi M, Michoud G, Daffonchio D. Grapevine rootstocks shape underground 671 bacterial microbiome and networking but not potential functionality. Microbiome 2018;6:3.

672 44. Fernández-González AJ, Cardoni M, Gómez-Lama Cabanás C, Valverde-Corredor A, Villadas PJ, 673 Fernández-López M, et al. Linking belowground microbial network changes to different tolerance level 674 towards Verticillium wilt of olive. Microbiome 2020;8:1-19.

675 45. Berry D, Widder S. Deciphering microbial interactions and detecting keystone species with co676 occurrence networks. Front Microbiol 2014;5:1-14.

677 46. Yun J-H, Roh SW, Whon TW, Jung M-J, Kim M-S, Park D-S, et al. Insect gut bacterial diversity 678 determined by environmental habitat, diet, developmental stage, and phylogeny of host. Appl Environ 679 Microbiol 2014;80:5254-64.

680 47. Jeon H, Park S, Choi J, Jeong G, Lee S-B, Choi Y, et al. The intestinal bacterial community in the 681 food waste-reducing larvae of Hermetia illucens. Curr Microbiol 2011;62:1390-9.

682 48. Varotto Boccazzi I, Ottoboni M, Martin E, Comandatore F, Vallone L, Spranghers T, et al. A survey 683 of the mycobiota associated with larvae of the black soldier fly (Hermetia illucens) reared for feed 
684 production. PLoS One 2017;12:e0182533.

685 49. Zhou J, Chen X, Yan J, You K, Yuan Z, Zhou Q, et al. Brummer-dependent lipid mobilization 686 regulates starvation resistance in Nilaparvata lugens. Arch Insect Biochem Physiol 2018;99:e21481.

687 50. Moghadam NN, Holmstrup M, Manenti T, Mouridsen MB, Pertoldi C, Loeschcke V. The role of 688 storage lipids in the relation between fecundity, locomotor activity, and lifespan of Drosophila 689 melanogaster longevity-selected and control lines. PLoS One 2015;10:1-18.

690 51. Zheng H, Powell JE, Steele MI, Dietrich C, Moran NA. Honeybee gut microbiota promotes host 691 weight gain via bacterial metabolism and hormonal signaling. Proc Natl Acad Sci USA 2017;114:477569280.

693 52. Chaston JM, Dobson AJ, Newell PD, Douglas AE. Host genetic control of the microbiota mediates 694 the drosophila nutritional phenotype. Appl Environ Microbiol 2016;82:671-9.

695 53. Chandler JA, Morgan Lang J, Bhatnagar S, Eisen JA, Kopp A. Bacterial communities of diverse 696 drosophila species: ecological context of a host-microbe model system. PLoS Genet 2011;7:e1002272. 697 54. Meneguz M, Schiavone A, Gai F, Dama A, Lussiana C, Renna M, et al. Effect of rearing substrate 698 on growth performance, waste reduction efficiency and chemical composition of black soldier fly 699 (Hermetia illucens) larvae. J Sci Food Agric 2018;98:5776-84.

700 55. Corby-Harris V, Pontaroli AC, Shimkets LJ, Bennetzen JL, Habel KE, Promislow DEL. 701 Geographical distribution and diversity of bacteria associated with natural populations of Drosophila 702 melanogaster. Appl Environ Microbiol 2007;73:3470-9.

703 56. Diamond J. Guns, germs, and steel: the fates of human societies. (W. W. Norton, 1999).

704 57. Vogel H, Müller A, Heckel DG, Gutzeit H, Vilcinskas A. Nutritional immunology: Diversification 705 and diet-dependent expression of antimicrobial peptides in the black soldier fly Hermetia illucens. Dev 706 Comp Immunol 2018;78:141-8.

707 58. de Vries FT, Griffiths RI, Bailey M, Craig H, Girlanda M, Gweon HS, et al. Soil bacterial networks 
are less stable under drought than fungal networks. Nat Commun 2018;9:3033.

709 59. Coyte KZ, Schluter J, Foster KR. The ecology of the microbiome: Networks, competition, and 710 stability. Science 2015;350:663-6.

711 60. Rojo D, Méndez-García C, Raczkowska BA, Bargiela R, Moya A, Ferrer M, et al. Exploring the 712 human microbiome from multiple perspectives: Factors altering its composition and function. FEMS

713 Microbiol Rev 2017;41:453-78.

714 61. Neilson JW, Califf K, Cardona C, Copeland A, van Treuren W, Josephson KL, et al. Significant 715 impacts of increasing aridity on the arid soil microbiome. mSystems 2017;2:1-15.

716 62. Callegari M, Jucker C, Fusi M, Leonardi MG, Daffonchio D, Borin S, et al. Hydrolytic profile of the 717 culturable gut bacterial community associated with Hermetia illucens. Front Microbiol 2020;11.

718 63. Ringø E, Hoseinifar SH, Ghosh K, Doan H Van, Beck BR, Song SK. Lactic acid bacteria in finfish719 an update. Front Microbiol 2018;9:1-37.

720 64. Yu G, Cheng P, Chen Y, Li Y, Yang Z, Chen Y, et al. Inoculating poultry manure with companion 721 bacteria influences growth and development of black soldier fly (Diptera: Stratiomyidae) larvae. Environ 722 Entomol 2011;40:30-5.

723 65. Zhan S, Fang G, Cai M, Kou Z, Xu J, Cao Y, et al. Genomic landscape and genetic manipulation of 724 the black soldier fly Hermetia illucens, a natural waste recycler. Cell Res 2020;30:50-60.

725 66. Cammack JA, Tomberlin JK. The impact of diet protein and carbohydrate on select life-history traits 726 of the black soldier fly Hermetia illucens (L.) (Diptera: Stratiomyidae). Insects 2017;8:56.

727 67. Danieli, Lussiana, Gasco, Amici, Ronchi. The Effects of Diet Formulation on the Yield, Proximate 728 composition, and fatty acid profile of the black soldier fly (Hermetia illucens L.) prepupae intended for 729 animal feed. Animals 2019;9:178.

730 68. Harris E V., de Roode JC, Gerardo NM. Diet-microbiome-disease: Investigating diet's influence on 731 infectious disease resistance through alteration of the gut microbiome. PLOS Pathog 2019;15:e1007891. 
69. Prosdocimi EM, Mapelli F, Gonella E, Borin S, Crotti E. Microbial ecology-based methods to characterize the bacterial communities of non-model insects. J Microbiol Methods 2015;119:110-25. primer sets for use in automated ribosomal intergenic spacer analysis of complex bacterial communities. Appl Environ Microbiol 2004;70:6147-56.

71. Kuczynski J, Lauber CL, Walters W a, Parfrey LW, Clemente JC, Gevers D, et al. Experimental and 738 analytical tools for studying the human microbiome. Nat Rev Genet 2012;13:47-58.

72. Mapelli F, Marasco R, Fusi M, Scaglia B, Tsiamis G, Rolli E, et al. The stage of soil development modulates rhizosphere effect along a High Arctic desert chronosequence. ISME J 2018;12:1188-98. analysis of high- throughput community sequencing data. Nat Methods 2010;7:335-6.

74. Edgar RC. UPARSE: highly accurate OTU sequences from microbial amplicon reads. Nat Methods 2013;10:996-8.

75. Ramette A. Multivariate analyses in microbial ecology. FEMS Microbiol Ecol 2007;62:142-60. Bioinformatics 2012;13:113.

77. Wang Y, Naumann U, Wright ST, Warton DI. mvabund - an R package for model-based analysis of multivariate abundance data. Methods Ecol Evol 2012;3:471-4. 
756 81. Hammer Ø, Harper DAT a. T, Ryan PD. PAST: Paleontological statistics software package for 757 education and data analysis. Palaeontol Electron 2001;4:1-9.

758 82. Wickham H. ggplot2: elegant graphics for data analysis. Media. Springer; 2016.

759 83. Faust K, Raes J. CoNet app: inference of biological association networks using Cytoscape. $760 \quad$ F1000Research. 2016;5:1519.

761 84. Marasco R, Mosqueira MJ, Fusi M, Ramond J, Merlino G, Booth JM, et al. Rhizosheath microbial 762 community assembly of sympatric desert speargrasses is independent of the plant host. Microbiome $763 \quad 2018 ; 6: 215$.

764 85. Doncheva NT, Assenov Y, Domingues FS, Albrecht M. Topological analysis and interactive 765 visualization of biological networks and protein structures. Nat Protoc 2012;7:670-85.

766 86. Bastian M, Heymann S, Jacomy M. Gephi: an open source software for exploring and manipulating 767 networks. Third Int AAAI Conf Weblogs Soc Media 2009;8:361-2.

768 87. Team RC, R Development Core Team. R: A language and environment for statistical computing. R 769 Found Stat Comput Vienna, Austria; 2013.

770 88. O’Hara RB, Kotze DJ. Do not log-transform count data. Methods Ecol Evol 2010;1:118-22. 
774 Table 1. Substrate consumption and developmental performances of FN- and NR-fed BSF individuals.

\begin{tabular}{|c|c|c|c|c|}
\hline \multirow{2}{*}{ Measurement } & & \multicolumn{3}{|l|}{ Diet } \\
\hline & & FN & NRF & NRV \\
\hline \multicolumn{2}{|c|}{ Diet energy (kcal/g) } & 1.62 & $0.38(-77 \%)$ & $0.19(-88 \%)$ \\
\hline \multirow{4}{*}{ Consumption } & Substrate consumption (\%) & $60.5 \pm 4.0(\mathrm{a})$ & $86.3 \pm 0.7(b)$ & $91.1 \pm 0.9(\mathrm{~b})$ \\
\hline & kcal/day/larva & $0.111 \pm 0.006$ (a) & $0.023 \pm 0.0004(b)$ & $0.008 \pm 0.0008(\mathrm{c})$ \\
\hline & ECD & $0.29 \pm 0.02(\mathrm{a})$ & $0.04 \pm 0.001(\mathrm{~b})$ & $0.08 \pm 0.01(\mathrm{c})$ \\
\hline & Waste reduction index & $4.22 \pm 0.11(\mathrm{a})$ & $1.04 \pm 0.01(\mathrm{~b})$ & $1.63 \pm 0.02(\mathrm{c})$ \\
\hline \multirow{4}{*}{$\begin{array}{l}\text { Developmental } \\
\text { stages }\end{array}$} & Larval development time* & $16 \pm 2(a)$ & $66 \pm 19(b)$ & $54 \pm 2(b)$ \\
\hline & Larval survival to prepupal stage & $94 \pm 5$ (a) & $94 \pm 1$ (a) & $92 \pm 7$ (a) \\
\hline & Adult emergence ${ }^{\#}$ & $92 \pm 7(\mathrm{a})$ & $73 \pm 2(\mathrm{~b})$ & $47 \pm 2(\mathrm{c})$ \\
\hline & Total survival $^{¥}$ & $86 \pm 4(a)$ & $68 \pm 2(b)$ & $44 \pm 4(\mathrm{c})$ \\
\hline
\end{tabular}

775

776

777

778

779

780

781

782

783

784

785

786

787

788

789
*Time from egg hatching to $40 \%$ of prepupal stage (days) obtained from three replicates; ${ }^{*}$ to prepupal stage; ${ }^{*}$ from prepupal stage; ${ }^{*}$ young larvae-adults. ${ }^{* *}$ Data were taken from [31].

Values are reported as average \pm standard deviation. Lowercase letters indicate results of Tukey's multiple comparison tests among diets.

BSF, black soldier fly; FN, full nutrient, NR, nutrient restriction; NRF, fruit NR; NRV, vegetable NR; ECD, efficiency of conversion of ingested food.

Table 2. Topological indices describing the co-occurrence networks of the entire life span of FN-, NRF-, and NRV-fed BSFs.

\begin{tabular}{lccc}
\hline \multirow{2}{*}{ Topological indices } & \multicolumn{3}{c}{ Diet } \\
\cline { 2 - 4 } & FN & NRF & NRV \\
\hline Number of nodes & 81 & 140 & 166 \\
Number of interactions & 1011 & 1129 & 1505 \\
$\quad$ Mutualistic & $622(62 \%)$ & $1004(89 \%)$ & $1107(74 \%)$ \\
$\quad$ Antagonistic & $389(38 \%)$ & $125(11 \%)$ & $398(26 \%)$ \\
Mean of degree \pm St. Dev. & $25 \pm 11 \mathrm{a}$ & $16 \pm 9 \mathrm{~b}$ & $18 \pm 12 \mathrm{~b}$ \\
\hline Cluster coefficient* & 0.58 & $0.67(+16 \%)$ & $0.59(+2 \%)$ \\
Centralization* & 0.35 & $0.15(-58 \%)$ & $0.2(-44 \%)$ \\
Average path length* & 1.77 & $2.61(+47 \%)$ & $2.46(+39 \%)$ \\
Average neighbors* & 24.96 & $16.13(-35 \%)$ & $18.13(-27 \%)$ \\
Density* & 0.31 & $0.12(-63 \%)$ & $0.11(-65 \%)$ \\
Heterogeneity* & 0.45 & $0.58(+30 \%)$ & $0.66(+47 \%)$ \\
\hline
\end{tabular}

*Percentage of increment (+) and decrement (-) in NRF and NRV with respect to FN are reported in brackets.

St. Dev., standard deviation; BSF, black soldier fly; FN, full nutrient; NRF, nutrient restriction fruit; NRV, nutrient restriction vegetable. 
792 Figure 1. Influence of diet influence on BFS growth and development. (a) Chemical composition of 793 the rearing substrates: FN, full nutrient; NRF, nutrient restriction fruit; NRV, nutrient restriction 794 vegetable. Carb, carbohydrate. (b) Larval growth rate; the fresh weight of larvae (FW) fed with FN, NRF, and NRV diets are reported as a function of time (days of growth). The correlation coefficient $\left(\mathrm{R}^{2}\right)$ of these two variables (FW and days) is reported for each diet in the graph; all correlations show significance probability, $p<0.0001$. (c and d) FW of larvae and pupae and (e) dry weight of adults are presented as average \pm standard deviation for the three diets (FN, NRF, and NRV). Weight is expressed in grams (g). Lowercase letters indicate the results of the Tukey's multiple comparison tests among the diets $800 \quad$ (significance, $p<0.05$ ).

801

Figure 2. Bacterial diversity associated with BFS fed with different diets across the developmental life cycle. (a-c) Principal coordinates analysis ( $\mathrm{PCoA})$ based on the Bray-Curtis dissimilarity matrices of bacterial OTU tables. Each symbol corresponds to one sample of a given developmental stage (a: larvae, circle; b: pupae, diamond: c: adult, triangle) and their colors indicate the different diets (black, full nutrient [FN]; orange, nutrient restriction fruit [NRF]; green, nutrient restriction vegetable [NRV]).

807 Results of multivariate analysis (GLM, general linear model) were also reported for each developmental 808 stage. (d-f) At each stage, variations in within beta-diversity were measured as the distance from the 809 centroid of each diet regime (i.e., the average dissimilarity from individual samples to their group 810 centroid). The distribution of within beta-diversity for each diet regime was visualized using boxplots 811 (reported data: minimum, first quartile, median, third quartile, and maximum) in (d) larvae, (e) pupae, 812 and (f) adults. Different lowercase letters above each boxplot denote significant mean difference in 813 dispersion based on the pairwise Tukey's test at $p<0.05$. ANOVA results were also reported. (g-i) 
814 Components of beta-diversity (similarity, replacement, and richness difference). Triangular plots were

815 used to visualize the relationships among the pairs of individuals for each diet in (g) larvae, (h) pupae,

816 and (i) adults. Each point (FN, black; NRF, orange; NRV, green) represents a pair of samples within the

817 diet regime. Its position is determined by a triplet of values from the similarity, replacement, and richness

818 difference. In each triplet, the large central dots from which the lines start (black, orange, and green) are

819 the centroid of the points; the lines represent the mean values of the similarity, replacement, and richness 820 difference components.

821

822 Figure 3. Bacterial community composition according to BFS developmental stage and diet 823 conditions. (a) Relative abundance of the bacterial class across BSF developmental stages under the 824 three diets (FN, full nutrient; NRF, nutrient restriction fruit; and NRV, nutrient restriction vegetable). 825 Relative abundance is expressed as percentage of sequence frequency. (b) Ternary plots indicate the 826 distribution of the OTUs across the three diets (FN, NRF, and NRV). The size and position of the circles 827 indicate the relative abundance and affiliation, respectively, of the OTUs within the three diets, whereas 828 the color indicates their phylogenetic affiliation in terms of their bacterial class.

Figure 4. Interactomes of the bacterial communities associated with BSF individuals fed on

831 different nutritional regimes. (a-c) Topological indices (centralization, density, and heterogeneity) of 832 the co-occurrence networks calculated for each developmental stage (L, larvae; P, pupae; and A, adults) 833 and the entire life span of BSF based on their feeding conditions (black, full nutrient (FN); orange, 834 nutrient restriction fruit (NRF); green, nutrient restriction vegetable). Values are expressed as percentage 835 compared with FN conditions (normobiotic). (d) Visualization of the bacterial co-occurrence networks 836 of insects fed with FN, NRF, and NRV diets, considering the entire life span of BSF. Each node 837 represents different bacteria (OTUs) and each edge represents significant co-occurrence relationships 
838 (green, mutualistic and red, exclusion). Node size indicates the abundance of each bacterium, whereas 839 the color indicates its phylogenetic affiliation at the class level. The modularity values are also reported. 840 (e) For each diet, the nodes with more degrees and higher betweenness centrality are classified as hubs 841 (black dots with gray border); among these, those in the first quartile are classified as keystones (black 842 dots with red border). Taxonomic affiliation of keystone nodes is also reported in pie charts. 

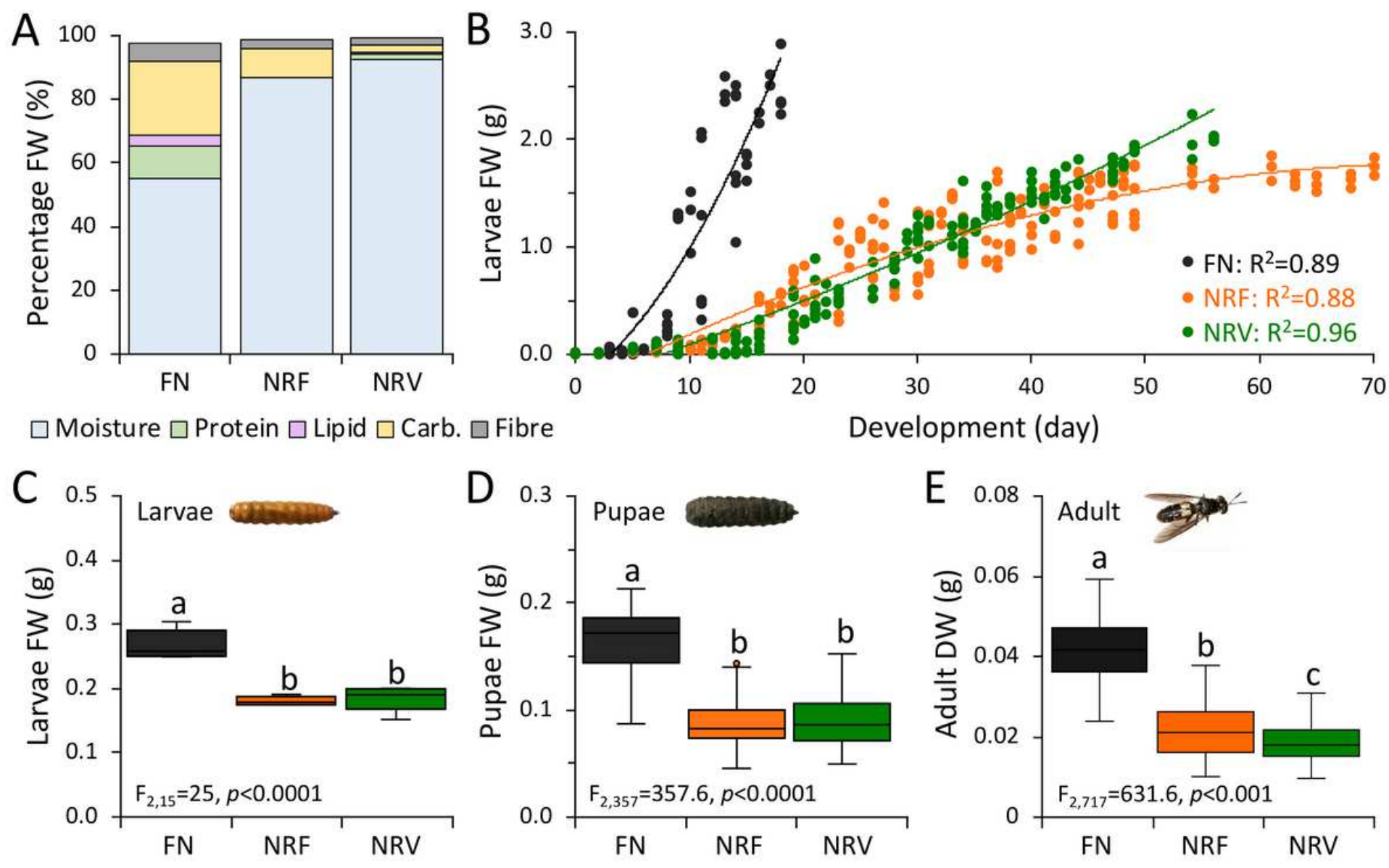

\section{Figure 1}

Influence of diet influence on BFS growth and development. (a) Chemical composition of the rearing substrates: FN, full nutrient; NRF, nutrient restriction fruit; NRV, nutrient restriction vegetable. Carb, carbohydrate. (b) Larval growth rate; the fresh weight of larvae (FW) fed with FN, NRF, and NRV diets are reported as a function of time (days of growth). The correlation coefficient (R2) of these two variables (FW and days) is reported for each diet in the graph; all correlations show significance probability, $\mathrm{p}<$ 0.0001 . (c and d) FW of larvae and pupae and (e) dry weight of adults are presented as average \pm standard deviation for the three diets (FN, NRF, and NRV). Weight is expressed in grams (g). Lowercase letters indicate the results of the Tukey's multiple comparison tests among the diets (significance, $p<$ 0.05). 

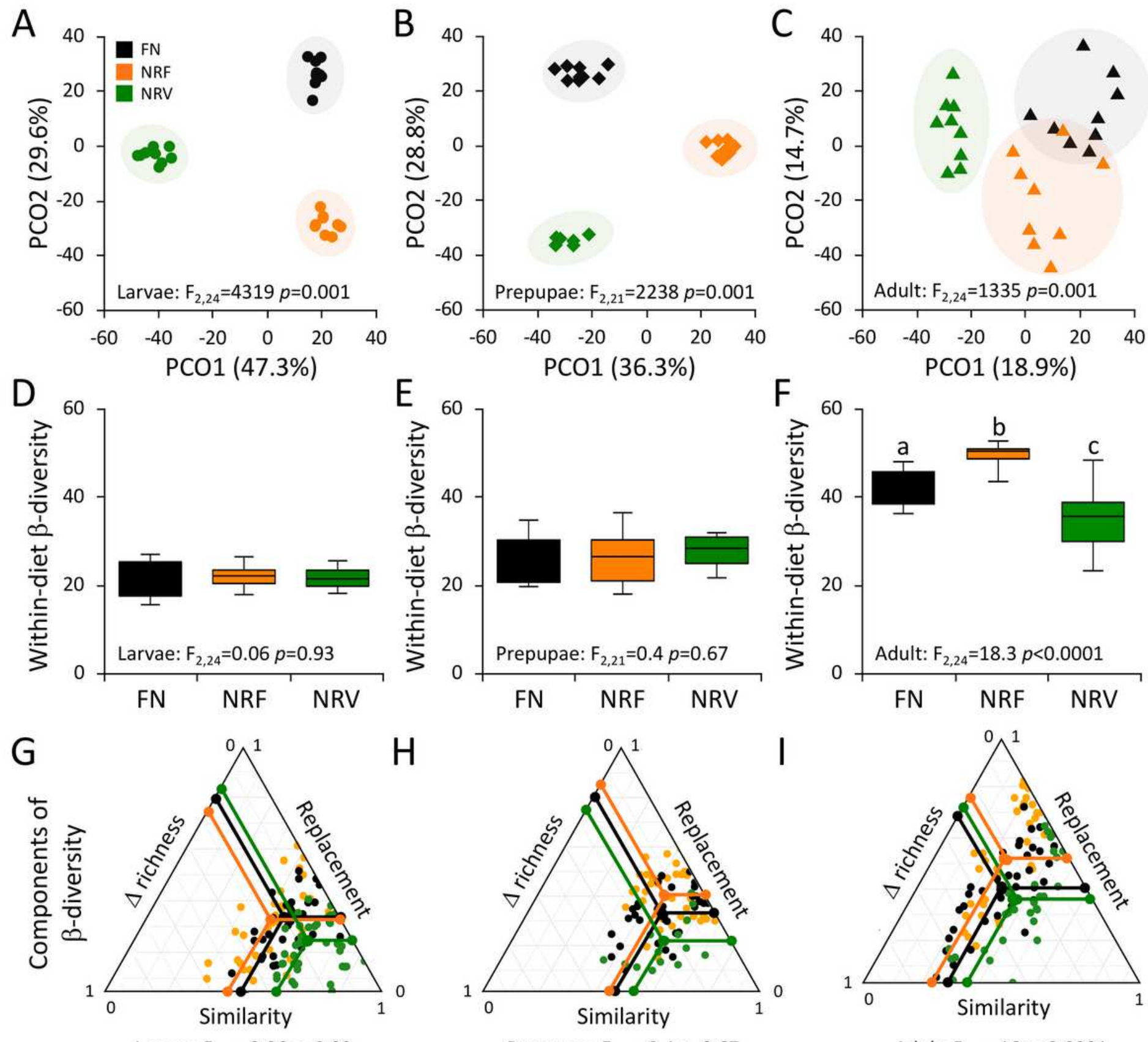

Larvae: $F_{2,24}=0.06 p=0.93$

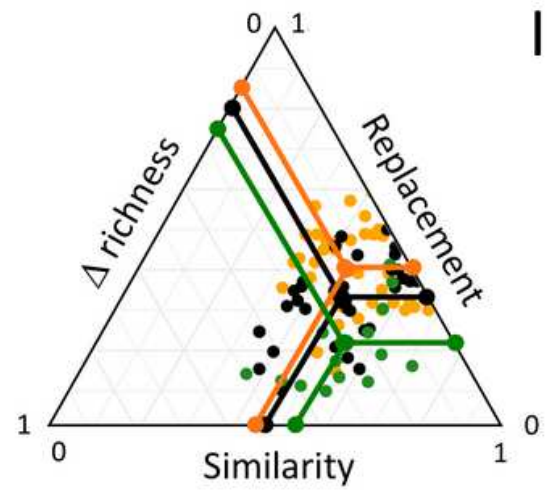

Prepupae: $F_{2,21}=0.4 p=0.67$

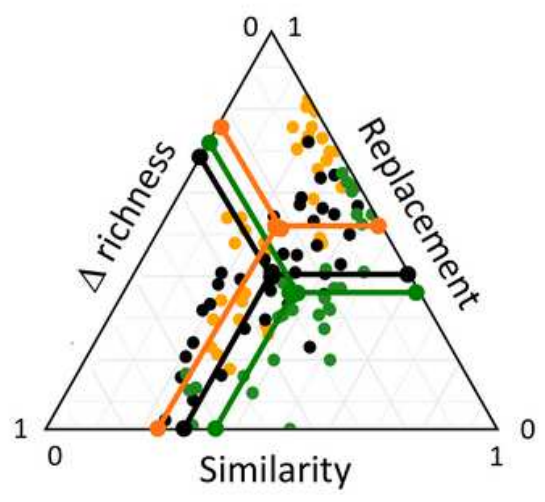

Adult: $F_{2,24}=16 p<0.0001$

Figure 2

Bacterial diversity associated with BFS fed with different diets across the developmental life cycle. (a-c) Principal coordinates analysis (PCOA) based on the Bray-Curtis dissimilarity matrices of bacterial OTU tables. Each symbol corresponds to one sample of a given developmental stage (a: larvae, circle; b: pupae, diamond: c: adult, triangle) and their colors indicate the different diets (black, full nutrient [FN]; orange, nutrient restriction fruit [NRF]; green, nutrient restriction vegetable [NRV]). Results of multivariate analysis (GLM, general linear model) were also reported for each developmental stage. (d-f) At each stage, variations in within beta-diversity were measured as the distance from the centroid of each diet regime (i.e., the average dissimilarity from individual samples to their group centroid). The distribution of within beta-diversity for each diet regime was visualized using boxplots (reported data: minimum, first 
quartile, median, third quartile, and maximum) in (d) larvae, (e) pupae, and (f) adults. Different lowercase letters above each boxplot denote significant mean difference in dispersion based on the pairwise Tukey's test at $p<0.05$. ANOVA results were also reported. $(\mathrm{g}-\mathrm{i})$ Components of beta-diversity (similarity, replacement, and richness difference). 814 Triangular plots were used to visualize the relationships among the pairs of individuals for each diet in ( $\mathrm{g}$ ) larvae, (h) pupae, and (i) adults. Each point (FN, black; NRF, orange; NRV, green) represents a pair of samples within the diet regime. Its position is determined by a triplet of values from the similarity, replacement, and richness difference. In each triplet, the large central dots from which the lines start (black, orange, and green) are the centroid of the points; the lines represent the mean values of the similarity, replacement, and richness difference components.

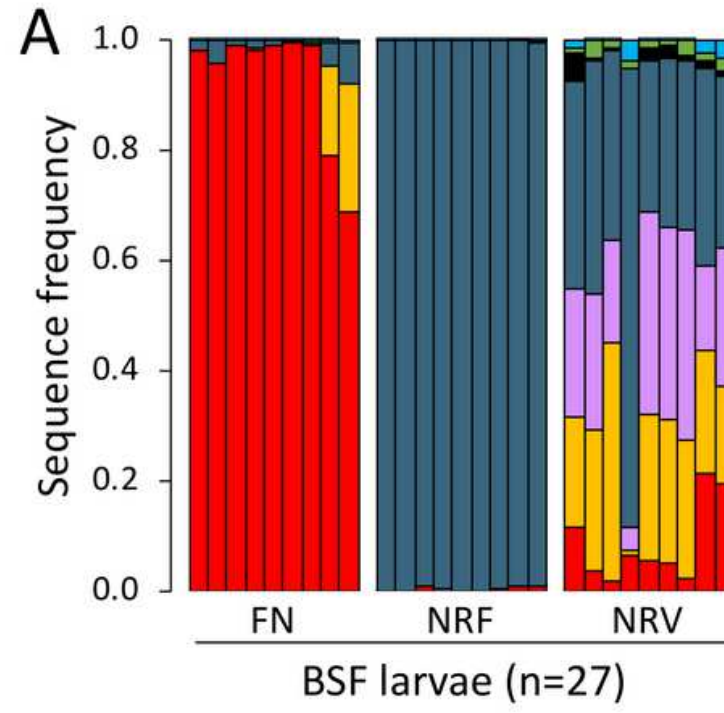

Bacilli $\square$ Bacteroidia

B

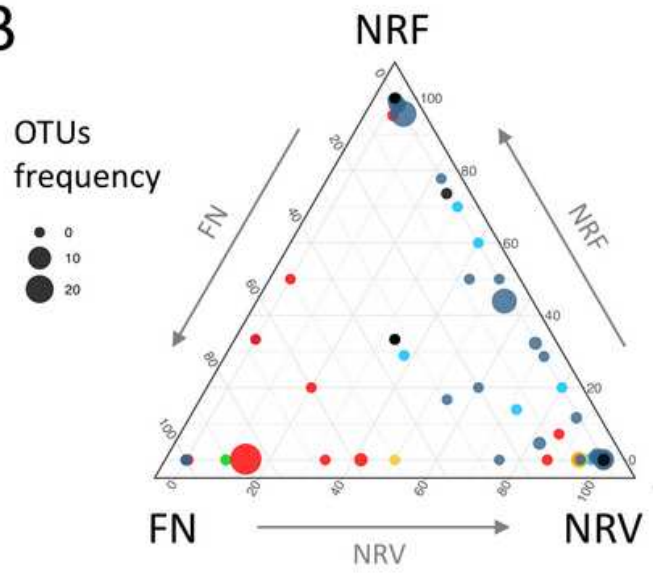

BSF larvae (OTUs=228)

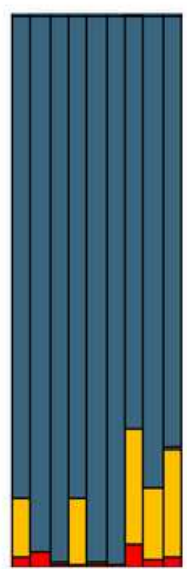

FN

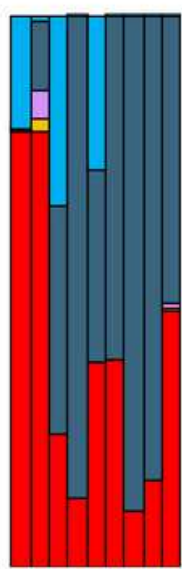

FN

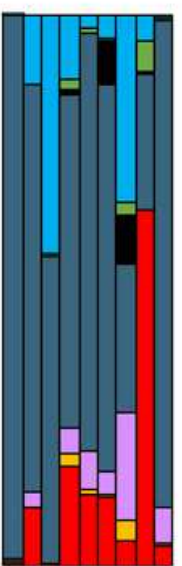

NRF

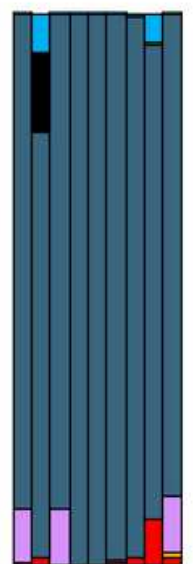

NRV

BSF prepupae $(n=24)$ BSF adult $(n=27)$

$\square$ Clostridia $\square$ Gamma $\quad$ Other

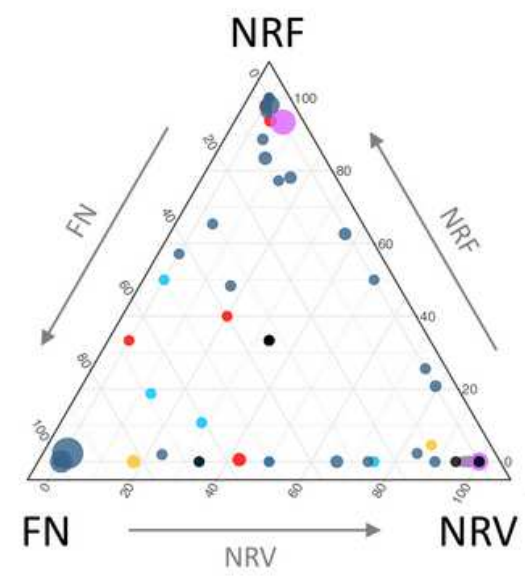

BSF prepupae (OTUs=179)

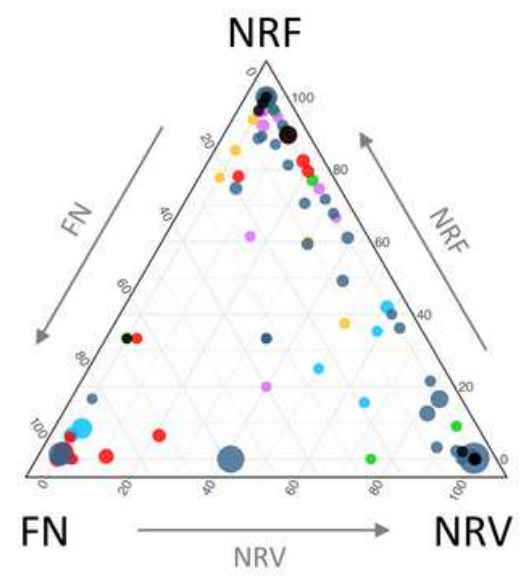

BSF adult (OTUs=225)

\section{Figure 3}

Bacterial community composition according to BFS developmental stage and diet conditions. (a) Relative abundance of the bacterial class across BSF developmental stages under the three diets (FN, full nutrient; NRF, nutrient restriction fruit; and NRV, nutrient restriction vegetable). Relative abundance is expressed as percentage of sequence frequency. (b) Ternary plots indicate the distribution of the OTUs across the three 
diets (FN, NRF, and NRV). The size and position of the circles indicate the relative abundance and affiliation, respectively, of the OTUs within the three diets, whereas the color indicates their phylogenetic affiliation in terms of their bacterial class.
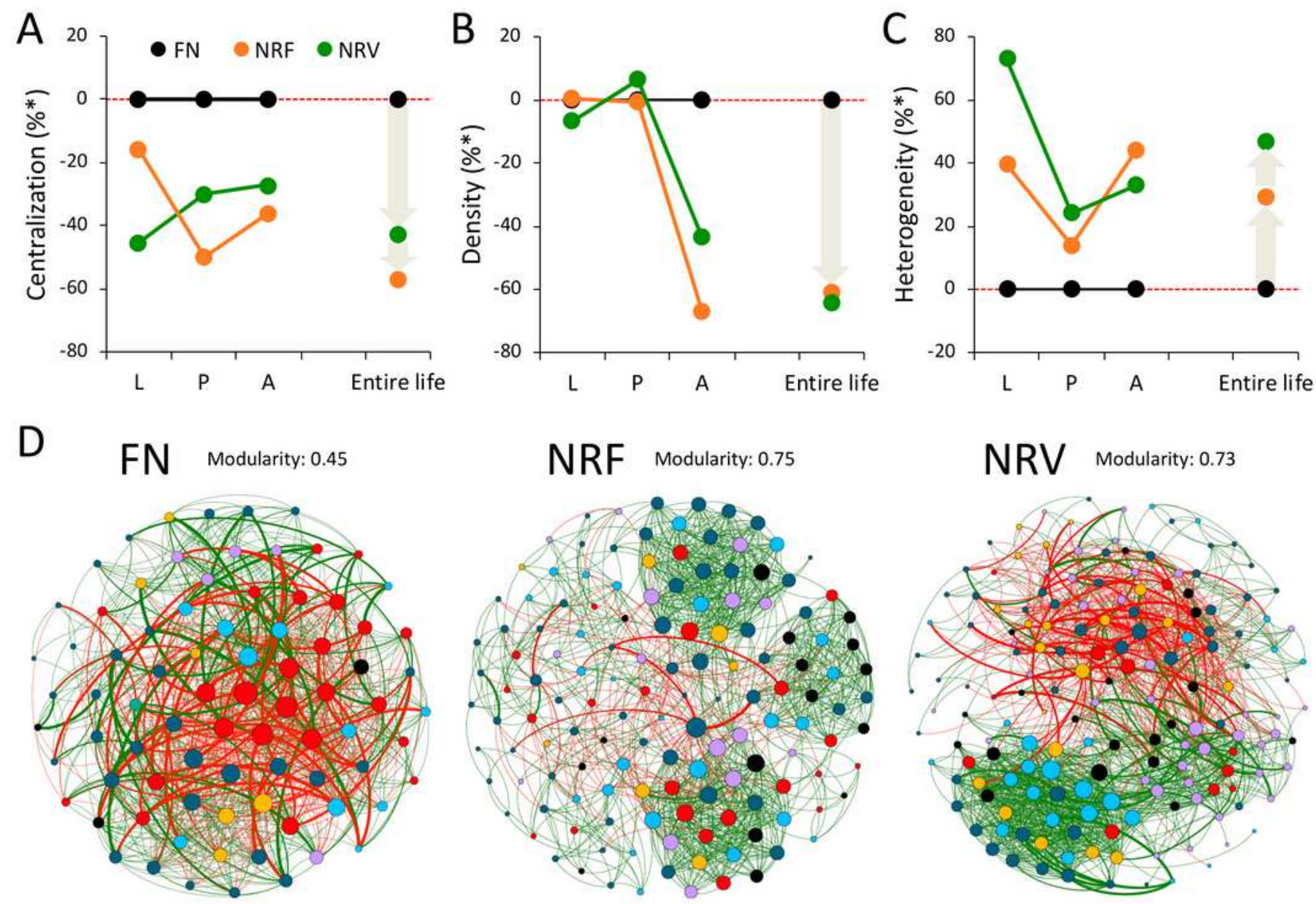

—Other $\square$ Alphaproteobacteria $\square$ Bacilli $\square$ Bacteroidia $\square$ Clostridia $\square$ Gammaproteobacteria
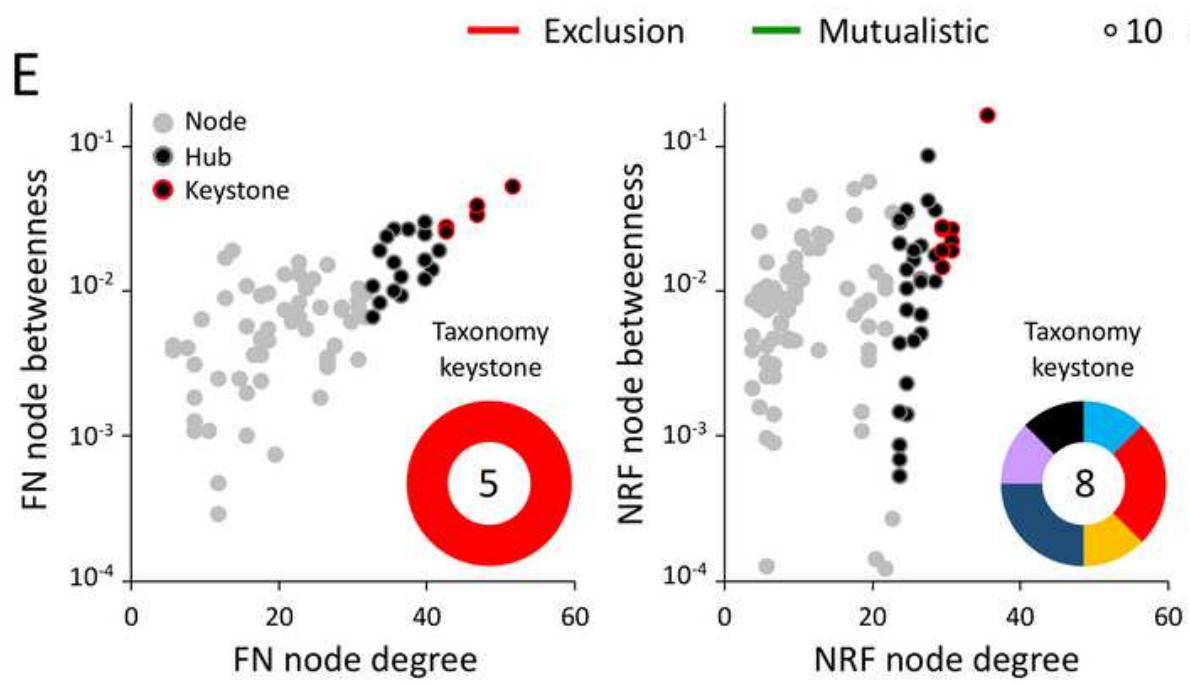

O 50

\section{Figure 4}

Interactomes of the bacterial communities associated with BSF individuals fed on different nutritional regimes. (a-c) Topological indices (centralization, density, and heterogeneity) of the co-occurrence 
networks calculated for each developmental stage (L, larvae; $P$, pupae; and $A$, adults) and the entire life span of BSF based on their feeding conditions (black, full nutrient (FN); orange, nutrient restriction fruit (NRF); green, nutrient restriction vegetable). Values are expressed as percentage compared with FN conditions (normobiotic). (d) Visualization of the bacterial co-occurrence networks of insects fed with FN, NRF, and NRV diets, considering the entire life span of BSF. Each node represents different bacteria (OTUs) and each edge represents significant co-occurrence relationships (green, mutualistic and red, exclusion). Node size indicates the abundance 838 of each bacterium, whereas the color indicates its phylogenetic affiliation at the class level. The modularity values are also reported. (e) For each diet, the nodes with more degrees and higher betweenness centrality are classified as hubs (black dots with gray border); among these, those in the first quartile are classified as keystones (black dots with red border). Taxonomic affiliation of keystone nodes is also reported in pie charts.

\section{Supplementary Files}

This is a list of supplementary files associated with this preprint. Click to download.

- Marascoetal2020Additionalfile16TableS9.xlsx

- Marascoetal2020SI.pdf 